\title{
Nano-bio interfaces probed by advanced optical spectroscopy: From model system studies to optical biosensors
}

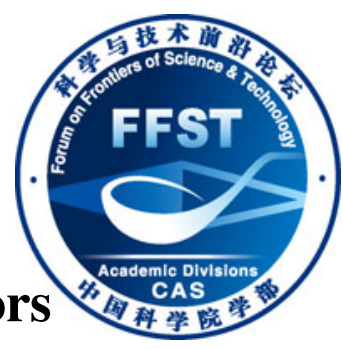

\author{
ZHANG XiaoXian, HAN XiaoFeng, WU FuGen, JASENSKY Joshua \& CHEN Zhan* \\ Department of Chemistry, University of Michigan, 930 North University Avenue, Ann Arbor, Michigan 48109, USA
}

Received September 16, 2012; accepted October 30, 2012; published online February 28, 2013

Research in biology and medicine is a rapidly expanding field incorporating some of the most fundamental questions concerning structure, function, and purpose. The forefront of new research demands access to advanced techniques and instrumentation capable of probing these unanswered questions. Over the past several decades, nano-scale materials and devices ranging from quasione dimensional quantum dots to two dimensional graphene sheets have been engineered and have found applications in nano-bio imaging and spectroscopy. In this review, the incorporation of nanomaterials into three influential spectroscopic and microscopic techniques including fluorescence microscopy, surface plasmon resonance, and sum frequency generation will be introduced. Fluorescence imaging has visualized nanomaterials as compliments or replacements to comparable organic fluorphores, act as a quencher for FRET-based sensing, and serve as a nanoscaffold for molecular beacons. Their versatility in coating materials makes nanomaterials an excellent targeting molecule for any cellular macromolecule or structure. In addition to the targeting capabilities of nanomaterials in fluorescence imaging, surface plasmon resonance has incorporated nanomaterials for applications in signal enhancement, selectivity of target molecules, and the development of more refined and accurate detection. Functionalized nanoparticles enhance the capabilities of sum frequency generation vibrational spectroscopy by providing unique surface chemistry which alters target molecule interactions and orientations. In summary, the incorporation of nanomaterials has greatly enhanced the field of biology and medicine and has allowed for the continual advancement of not only research but instrument development.

nano-bio interfaces, optical spectroscopy, fluoroscence, SERS, SFG

Citation: Zhang X X, Han X F, Wu F G, et al. Nano-bio interfaces probed by advanced optical spectroscopy: From model system studies to optical biosensors. Chin Sci Bull, 2013, 58: 2537-2556, doi: 10.1007/s11434-013-5700-y

Nanomaterials, which size ranges $(1-100 \mathrm{~nm})$ coincide with fundamentally important length scales in physics, are considered to be promising building blocks for future electrical, optical and optoelectronic applications [1-8]. For example, in metals, the mean free path of an electron at room temperature is $\sim 10-100 \mathrm{~nm}$ [9]; the Bohr radius of photo-excited electron-hole pairs in semiconductors is $~ 1-10 \mathrm{~nm}$ [10]; and the $1-100 \mathrm{~nm}$ dimension is the range over which molecules assemble into nucleic acids and proteins [11]. Nanostructures on this size scale provide a unique technology to determine many critical structure/function relationships of biological molecules at the cellular level without introduc-

*Corresponding author (email: zhanc@umich.edu) ing substantial interference.

Advances in nanotechnology have developed at several stages: materials, devices, and systems. A decade ago, extensive research has focused on the development of various methods to precisely control the synthesis of nanomaterials. Therefore, many different kinds of nanostructures were designed and synthesized, including quasi-zero dimensional (0D) nanodots (NDs) [12-14], quasi-one dimensional (1D) nanowires (NWs) [15-17], quasi-two dimensional (2D) nanosheets [18-21], and other complicated nanostructures [22-25]. Typically, metal and semiconductor NDs in the 2-6 nm size are of greatest interest, not only for their unique size-dependent physical and chemical properties but also their size similarities to biological molecules [12,13,26-28]. 
1D NWs are becoming a popular alternative in biological labeling to quantum dots due to their capability of providing polarized emission, reduced blinking, and faster radioactive rates [29-32]. Recently, commonly-used NDs and NWs, 2D nanosheets, especially graphene and graphene oxide (GO) [33-36], are utilized as nano-bio probes due to their large surface areas, atomic level thicknesses, and easy chemical modification. Here, especially in Section 1 (Fluorescence), we will focus on the fluorescent studies of optical sensing based on above-mentioned nanostructures (e.g. NPs, NWs and nanosheets-like graphene and GO). Since various nanostructures can be successfully synthesized and precisely controlled, we believe that the development of nanotechnology can transition towards the construction of new nanodevices, and eventually, functional nanosystems [37].

Over the past decade, many research groups all over the world have developed unique and versatile applications based on nanomaterials. Among them, the integration of these materials with biology and medicine has shown substantial progress and excellent results have been generated from such nano-bio studies. The literature on the bio-nano interaction and its applications is growing rapidly; mainly including studies on (1) fundamental mechanisms governing bio-nano interactions and (2) bio-detection applications. Some recent reviews have systematically summarized significant developments in understanding the interactions at the nano-bio interface as well as some applications on nanobio probe-based electric and magnetic field detection $[38,39]$. These methods that have been reviewed by other groups will not be covered in detail here. In this review, we will present recent research progress in nanomaterial-based biological detection using advanced optical spectroscopic techniques. Three important optical methods, including fluorescent microscopy, surface plasma resonance (SPR) and sum frequency generation (SFG) vibrational spectroscopy are discussed here.

During the past 30 years, there has been remarkable growth in the use of fluorescence in the biological sciences and medical community. Fluorescence microscopy, known for its versatility and specificity, has relied on organic fluorphores, fluorescent proteins (such as GFP and its derivatives), and various autofluorescent biomolecules. For examples, 4',6-diamidino-2-phenylindole (DAPI) [40,41] and Hoechst dyes [42,43] are utilized extensively in the imaging of nuclei since they bind to the minor groove of DNA. Organic and biological probes, although very useful, only allow for the observation of specific structures which have been fluorescently labeled. This, however, may lead to the missing information within unlabeled portions of the sample. To address this issue, label-free optical sensing is found to be an attractive alternative, not only because of its capability of detecting biomolecules without using any radioactive or fluorescent labels, but also its same selectivity and sensitivity as its biological counterparts. Such labels usually introduce complexity and potential contaminations into the biological systems in vivo.

Surface plasmon resonance (SPR) is one of the most important label-free methods for highly sensitive probing of biomolecules [44-46]. SPR can be described as the resonant oscillation of surface electrons (usually in a metal) stimulated by incident light under a resonance condition. During this process, surface electromagnetic waves, i.e. surface plasmon polaritons, propagate in the direction parallel to the metal/dielectric interface. These oscillations are very sensitive to any change in the surface refractive index and can detect adsorption and binding events of molecules on a metal surface [45]. Particularly, nanosized novel metal particles or nanowires, which can exhibit strong absorption bands in the ultraviolet to visible region [47], are extensively used to probe biomolecules such as DNA and proteins [48-51].

Sum frequency generation (SFG) vibrational spectroscopy, a second order nonlinear optical spectroscopic technique, is a powerful tool to provide chemical and structural information of molecules on surfaces and at buried interfaces [52-54]. Recently, SFG has been widely applied to study important subjects in biology and the life sciences, such as lipids, peptides, proteins, and DNA [55-80]. In this review (Section 3), we will briefly review SFG studies on model cell membranes, then overview the recent progress of nanoparticle-related system research, and finally provide insight into its potential applications on nano-bio systems.

As details of nanomaterials and analytical methods have been reviewed elsewhere, this review highlights the investigations on nano-bio interfaces using the previously discussed three techniques, and then provides an overview of their integration into an emerging nano-bio and nanomedicine field. New developments on the nanomaterial applications in optical biosensing, existing problems, and future directions will also be discussed.

\section{Fluorescence}

To begin with, we will review the applications of the fluorescence technique in nanomaterials. We will introduce how to attach biomolecules to the surfaces of nanomaterials, and how fluorescence can be applied to detect nano-bio systems.

\subsection{Attachment of biomolecules to nanomaterials}

In order to probe biological molecules optically with nanomaterials, the first prerequisite is to understand the biophysical or biochemical interactions at nano-bio interfaces and then to demonstrate how to attach biological molecules to nanomaterials. The elucidation on the interactions at nano-bio interfaces can lead to the design of improved nano-bio probes. In 2009, Nel et al. [81] comprehensively reviewed the studies on interactions at the interfaces of nanoparticles and proteins, membranes, cells, DNA, and orga- 
nelles over the past decades. Herein, we will not cover above information but only briefly introduce the methodologies that have been commonly utilized to attach biological molecules to nanomaterials.

It is well known that certain biomolecules are capable of molecular recognition; typical associations are described under the "lock and key" principle common to most enzyme/ ligand recognition. Receptor molecules (just like locks) can recognize certain ligand molecules (just like keys) with very high specificity. Only a specific ligand can bind to its receptor, such as antibodies binding to antigens, oligonucleotides binding to their complementary counterparts, and the biotin-avidin system [82]. The underlying mechanism of molecular recognition is essential to facilitate biomolecule and nanomaterial interactions.

Generally, there are two ways to assemble biomolecules with nanomaterials: one is direct attachment of the biomolecule to the nanomaterial surface using a ligand exchange. For instance, phosphine molecules which are used as a stabilizing shell around gold NPs, can be fully or partially replaced by thoil group modified oligonucleotides, thus single stranded oligonucleotides of different lengths can be attached to the surface of these gold NPs with different DNAto-gold ratios $[83,84]$. The other method is to bind the biomolecules to the stabilizing shell around nanomaterial cores [26]. For example, in 1998, Chan et al. [26] attached biomolecules to mercaptoacetic acid modified semiconductor quantum dots via forming a covalent coupling between reactive amine groups and free carboxyl groups.

Various interactions can be employed to connect biomolecules and nanomaterials. In certain circumstances, the molecules are directly adsorbed to either the nanomaterial surface or the shell of stabilizing molecules around the nanomaterial [85]. For example, direct binding of fullerenespecific monoclonal antibodies to single walled carbon

(a) Bifunctional linkage

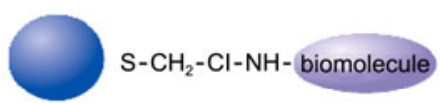

(b) Hydrophobic attraction

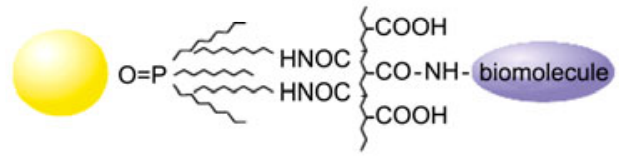

(c) Silanization

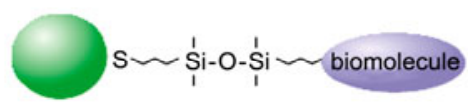

Current opinion in biotechnology nanotubes [86] and entropic effects driving adsorption of calf thymus DNA to CdS NPs [87] have been demonstrated as possible attachment methods. However, for both of these cases, stability is poor. Therefore, electrostatic interactions $[88,89]$, hydrophobic attraction [90,91] and formation of stronger chemical bonds [26,91], have been commonly utilized in connecting biomolecules and nanomaterials for their increased stability. In latter cases, biomolecules are usually oppositely charged to the nanomaterials, e.g. a chimeric fusion protein was designed to electrostatically bind to the oppositely charged surface of $\mathrm{CdSe} / \mathrm{ZnS}$ core/shell NPs [88]. The formation of chemical bonds is one of the most important methods for forming nano-bioconjugates. The above-mentioned thiol groups have been extensively used to assemble biomolecules to the surface of gold NPs via $\mathrm{Au}-\mathrm{S}$ bonding. Other bonds like $\pi-\pi$ interaction, covalent amide linkage, silanization, etc. also play important roles to prepare an essential bio-nano interface. Up to now, various flexible bioconjugation techniques with high reproducibility have been exploited and applied to nanomaterials (shown in Figure 1), which lead to substantial progress in the development of nano-biosensors and other potential biological applications.

\subsection{Nano-biofluorescence detection}

It is of great importance to realize the complex interplay of different biomolecules from the cellular to the integrative level. Hence, fluorescent labeling has been extensively used for both in vivo cellular imaging and in vitro assay detection [93]. The intrinsic optical properties of organic fluorphores and genetically expressed fluorescent proteins, however, usually have limited efficiency in long-term imaging and "multiplexing" due to their broad absorption/emission profiles and low photobleaching thresholds at room temperatures

(d) Electrostatic attraction

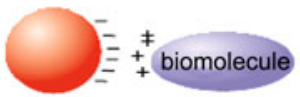

(e) Nanobeads

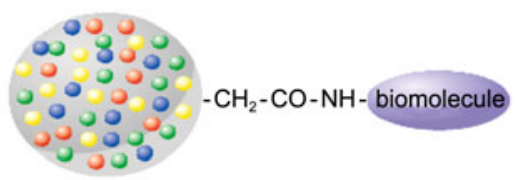

Figure 1 (Color online) Schematic illustrating various methods to attach biomolecules to nanomaterials. Reprinted from Ref. [92] under the permission of Elsevier. 
[94]. For this purpose, nanomaterials, especially quantum dots with tunable emission, high quantum yield, high molar extinction coefficients, broad absorption with narrow photoluminescence (PL) spectra from ultraviolet to near-infrared, high multiplexing capability, large effective Stokes shifts, high brightness as well as high resistance to photobleaching, have proven to be widely applicable and very appealing to the biological imaging field $[12,95]$.

Many biosensors constructed based on 1D NWs or 2D nanosheets, rely on the detection of electrical and electrochemical signals. 1D nanowires/carbon nanotubes and 2D graphene/GO exhibit remarkable electronic properties and own the flexibility and the facility of morphologies that can be easily microfabricated into electrical devices [38,96,97]. Reports of using nano devices for optical bio-detection, in particular, fluorescent labeling, imaging, and sensing demonstrate one of the many niches that these materials and devices are able to fulfill. Using NPs and NWs allows for real-time monitoring and tracking of intracellular processes on relevant biological time scales. Size-dependent PL emission shift in a wide range enables simultaneous imaging of multiple targets inside living cells or on the cell surface by multicolor QDs at the same time. As for in vivo cell imaging and the tracking of biomolecules, water-solubility, size similarity to fluorescent proteins, and excellent optical properties are three key points for researchers' consideration in choosing nanomaterials. Therefore, QDs have been integrated heavily in imaging research along with, more recently, quantum wires (QWs) as well as small-diameter carbon nanotubes. In recent years, researchers have also placed much emphasis into uses for graphene and GO, a large planar structure and single carbon atomic layer of which make them ideal substrates for the absorption of different kinds of biomolecules and cells simultaneously, thus enabling complicated biological process observed in situ. In this section, we selectively summary the recent research developments of fluorescent-based biosensors, and the relevant researches based on various nanomaterials from 0D NPs, 1D NWs as well as 2D graphene and GO.

(i) 0D Nanoparticles. Due to a tunable fluorescence emission profile over a broad range and ease of synthesis, semiconductors are commonly used for fluorescent detection. Past years of engineering work have focused on precisely controlling their size and shape, using CdSe and CdS as their material of choice. These particles have absorption profiles that can be tuned throughout the visible region (bandgaps are $1.7 \mathrm{eV}$ for $\mathrm{CdSe}$ and $2.4 \mathrm{eV}$ for $\mathrm{CdS}$ ), with direct proportionality to size versus adsorption (the smaller the particle, the shorter the absorption wavelength). For the reasons stated above, semiconductor nanoparticles are an ideal choice for fluorescence imaging applications; however these particles sometimes have practical limitations to their use. Surface defects caused by surface vacancy, lattice mismatch, dangling bonds, or absorbates at the surface, trap the excited electrons, or holes thus depressing the recombi- nation efficiency of luminescence, consequently weakening and even quenching the PL emission.

To minimize the effect of surface imperfections on the emission profile of these particles, surface passivation has been commonly used to protect the surface of NPs by coating another material with a larger bandgap in order to decrease the possibility of charge carriers trapped in surface defects and confine the excited electron and holes inside the NPs. For example, $\mathrm{ZnS}$ and amorphous $\mathrm{SiO}_{2}$ are commonly used as core-shell nanocomposite materials for $\mathrm{CdSe}$ and $\mathrm{Si}$ NPs. Notice that except for the reason listed above, the choice of the protection layer also depends on changing hydrophilicity and biocompatibility of NPs, thus enabling the formation of nano-bioconjugates of interest. For instance, an outer shell made of silica will make the NPs water soluble [98]. After effective surface passivation, the NPs can have quantum yields comparable to organic dyes, and more importantly, these particles become more biocompatible. This results in an excellent inorganic analogue to commonly used fluorescent dyes/proteins with much narrower emission, size-dependent tunability, and a strong resistance to photobleaching.

In 1998, both Alivisatos group [98] and Nie group [26] reported their success on QD-based specific fluorescent biological stains in cells for the first time. In both experiments, CdSe QDs of various sizes, which emitted at different wavelength maxima, were coated by a $\mathrm{CdS}$ [98] and $\mathrm{ZnS}$ [26] shell thus allowing for covalent attachment of protein molecules to the surface of NPs via chemical interaction. Specifically, Nie group [26] used mercaptoacetic acid for solubilization and covalent coupling to various biomolecules via cross-linking to reactive amine groups. While in the case of Alivisatos group, the avidin-biotin interaction, a model system for ligand-receptor binding, was utilized. Hence, incubating the protein-decorated QDs with different cells led to the specific fluorescent labeling of the cells; where the protein shell provided specificity while the semiconductor QDs core gave size-dependent luminescence profiles. Figure 2(a) gives clear evidence that decorated QDs penetrated into the nucleus (green color) [98], and Figure 2(b) and (c) indicate that fluorescence images could be obtained from cultured HeLa cells incubated with mercapto-QDs (Figure 2(b)). With transferrin-QD bioconjugates (Figure 2(c)), this comparison shows that no QDs were transported into the cells without first incubating with transferrin [26]. Moreover, Nie group [26] also studied the possibility of using QDs probes for immunoassay. Incubated with bovine setum albumin (BSA) and with a specific polyclonal antibody, fluorescence images of corresponding QD-immunoglobulin G ( $\mathrm{IgG}$ ) composites indicated that QD aggregated dramatically in the presence of IgG (Figure 2(e)), while BSA still remained well dispersed throughout the cell (Figure 2(d)).

With the incorporation of nanomaterials into biological imaging, many groups have developed various in vitro and 


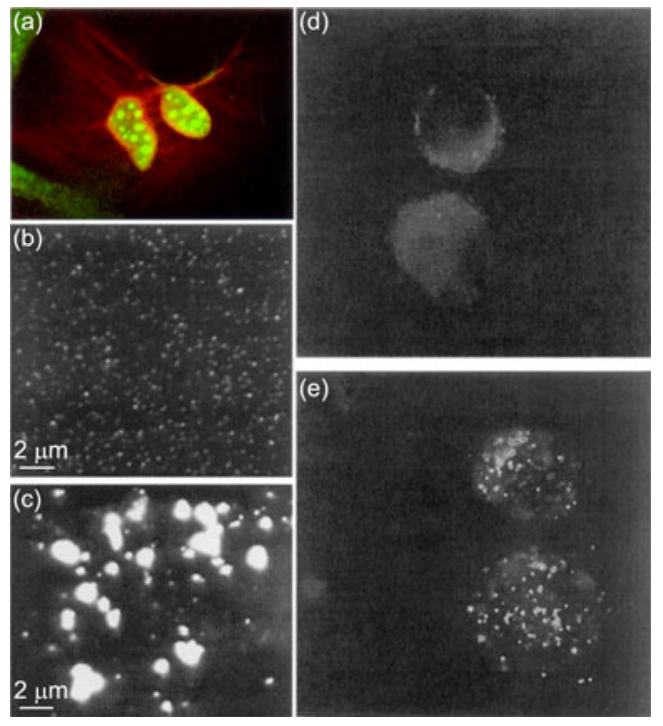

Figure 2 Fluorescence images of mouse 3T3 fibroblasts incubated with decorated QDs (a) (reprinted by permission of Science [98]), cultured HeLa cells incubated with mercapto-QDs (b) and QD-transferrin conjugates (c) (reprinted by permission of Science [26]). Luminescence images of QD conjugates with BSA (d) as well as aggregation of QD in the presence of a specific polyclonal antibody (e) (reprinted under permission of Science [26]).

in vivo applications through broad utility of QDs, such as cell labeling, tracking cell migration, fluorescence in situ hybridization, whole animal contrast agents, and Förster resonance energy transfer (FRET) sensors. Among them, the visualization and mapping of biomolecular networks that elucidate the function and viability of cells or even entire organs is an important application of QDs as molecular probes. Therefore, except for substitution of fluorescent dye molecules, NPs have also been employed as powerful optical probes for local biological environment due to their large surface/volume ratio. For example, Derfus et al. [99] reported that endocytosis of QDs into HeLa cells is a unique system which combines both biochemical (e.g. translocation peptides, cationic liposomes, and dendrimers) and physical processes (electroporation and microinjection). Significant localization and accumulation of QDs were clearly displayed after injection of peptide-decorated QDs (Figure 3(a)). Moreover, Gao et al. [100] also utilized $\mathrm{ZnS}$ capped CdSe QDs with three different surface modifications to target cancer cell in vivo. Figure 3(b) provides one example that elucidate the transportation of tumor cells inside a mouse body probed by prostate-specific membrane antigen (PSMA)-modified QDs.

Just as discussed above, the photoluminescent properties of semiconductors are very sensitive to local environment as well as the amount of adsorbates on the surface. Hence, QDs can and have been used to detect uncommon conformations in DNA. From 1995, the Murphy group [87,101,102] started to demonstrate a series of research results on DNA fluorescent detection via coupling DNA with CdS QDs. In these experiments, surfaces of QDs were decorated with

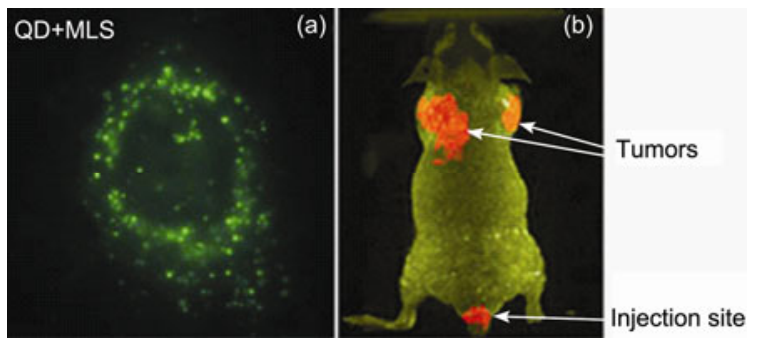

Figure 3 (a) Fluorescence micrographs of a HeLa cell $24 \mathrm{~h}$ after injection of mitochondrial localization sequence peptide-QDs. Reproduced under permission from Wiley [99]. (b) In vivo fluorescence images of a tumor-injected mouse using PSMA-QDs as probes. Reproduced under permission from Nature publishing group [100].

cationic or hydrogen-bonding groups in order to attract the anionic DNA. Subsequently, luminescence titrations were carried out with oligonucleotides by QDs to discriminate between different local structures and conformation, e.g. "straight", "bent", and "kinked" in dilute solution [101]. It is worthy nothing that the emission profile of these QDs only changed in intensity, and not the overall lineshape.

Additionally, Benoit and co-workers [90] demonstrated in vivo and in vitro studies of $\mathrm{ZnS}$-coated CdSe QDs encapsulated in phospholipid block-copolymer micelles. They found that these nanocrystal-micelle conjugates remained stable and were slow to photobleach once they were injected into Xenopus embryos. Most importantly, various DNA, even some that are related to human disease, can bind to this protein-sized surface without labeling the DNA with organic dyes or using radioactivity.

While semiconductor materials comprise the majority of NPs used for biosensing, some other materials such as metallic and polymer QDs have also been used as fluorescent probes. The most common uses for metal NPs still are applicable to areas such as surface plasmon resonance spectroscopy (SPR) and surface-enhanced Raman spectroscopy (SERS) (This part will be discussed in next section). Some groups reported their success on fluorescent sensing via nano-bio interaction. For instance, Taton et al. [103] modified the surface of the gold NPs with DNA sequences; by adding in complimentary DNA strands to solution, a visible color change was observed with femtomolar detection limits. Additional reports about "fluorescent" NPs are actually based on organic fluorescent dyes fluorescence but not NPs, so these results will not be discussed in detail here.

Except for individual NPs, well-patterned NPs that were capable of parallel integration for detection of multiple signals, emerged with the ability to sense the biomolecules at the single molecule level in living cells. Especially in the 1980s, Yablonovitch [104] first proposed "photonic crystals", an analogous concept to control the density of photon states in much the same way a semiconductor affects the properties of an electron. By enclosing a molecule in a welldesigned photonic crystal, it is possible to detect the molecules via controlling the rate and direction in which mole- 
cules emit light [105,106]. Asher and co-workers [106-108] reported a method of biological screening based on photonic crystals. In these measurements, polymerized crystalline colloidal arrays were applied to detect lead and carbohydrates in bodily fluids.

Although many researchers in recent years have developed various methods for QD-based biological detection via fluorescence, QDs have limitations including accurate control of particle size after coating. Toxicity of heavy metal elements such as $\mathrm{Cd}, \mathrm{Zn}, \mathrm{Hg}$, and $\mathrm{Pb}$ from commonly used QDs to animals and human medical applications in vivo is still a main concern. Therefore, many researchers believe that QDs are more likely to be a complement to existing organic fluorophores rather than a replacement.

(ii) 1D nanowires and 2D graphene/graphene oxide. Compared to quasi-zero dimensional NPs, NWs are also utilized to label biological molecules. Because of their unique electrical characteristic and the flexibility in their morphology for microfabrication, 1D semiconductor NWs as well carbon nanotubes (CNTs), have been used as biosensors based on electrical measurements. 1D and 2D nanostructures used for fluorescent detection are another important research in this field and this section will review the developments using 1D and 2D nanostructures as fluorescent bio-detection. Some examples of NW or CNT as well as graphene/GO based fluorescent bio-applications will be introduced herein.

Semiconductor NWs or nanorods, as a 1D structure, can provide polarized emission, and therefore, were thought to be promising building blocks for biological labeling [30,109]. Considering the size similarity to common fluorescent proteins such as GFP and its derivatives, QDs or rods (QRs) with diameter ranging from 2-10 nm and with length from $5-100 \mathrm{~nm}$ were primarily chosen for cell labeling and biosensing. In addition to the great optical properties inherent in QDs such as tunable emission, high resistance to photobleaching, and a narrow symmetric emission profile, QRs own some other unique properties that may lead to highly sensitive biological labels including reduced blinking and faster radiative rates. In 2001, the Alivisatos group [30] demonstrated the size- and shape-dependent bandgap variation of CdSe quantum rods, as well as analyzed their potential usage for bio-labeling. Subsequently, Alivisatos and co-workers [109] were able to modify the surface of CdSe QRs with CdS and $\mathrm{ZnS}$, allowing for their used in biological labeling. In this work, surface silanization was implemented to prepare water-soluble bio-QRs conjugates (shown in Figure 4). Then under the usage of streptolysin-O (SLO), a bacterial protein that commonly binds to cholesterol and forms holes in the plasma membrane of animal cells, a small amount of silanized QRs were transferred to human breast cancer cell line MDA-MB-231. QRs were found to retain their brightness inside living cells (Figure 4(b)). In comparison to fluorescence signals from QRs and QDs, much brighter signals were obtained by using QRs as single

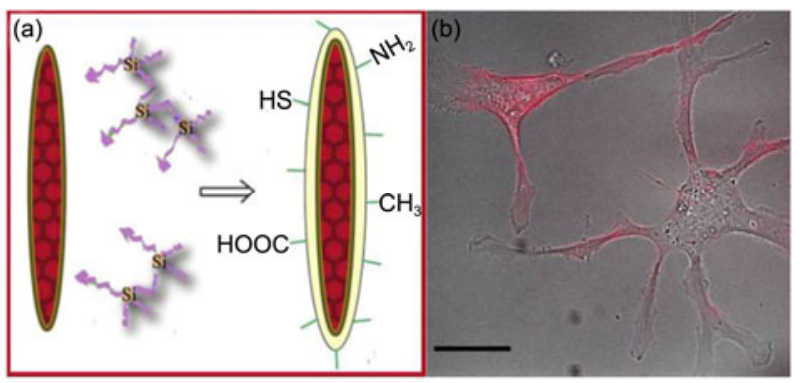

Figure 4 (a) A schematic illustrating surface silanization of quantum rods $(\mathrm{QR})$. (b) The merged images of transmission and fluorescent micrograms of bio-QRs conjugates in human breast cancer cells MDA-MB231. The red fluorescence in the images is from QRs in cells after $1 \mathrm{~h}$ transfected with Chariot. Scale bar is $20 \mu \mathrm{m}$. Reprinted with permission from [109]. Copyright 2007 American Chemical Society.

molecule probes.

Except for semiconductor QDs, carbon nanotubes (CNTs) have also been utilized as medical imaging tools. CNTs have distinguished factors such as high quantum efficiency, light emission in a very narrow range of wavelengths, the ability to produce light in the near infrared region where skin and other tissue is transparent, extreme resistance to degradation, and low damage to living cells. These characteristics make CNTs particularly well suited for use in living systems. In the past ten years, research has been devoted to CNT-based bio-detection, such as targeted delivery of chemotherapeutics, diagnostic contrast agents and photoablative therapy agents, and good cargo modules for DNA, siRNA, proteins and peptides [110-115]. Nevertheless, due to their excellent fluorescence properties, some attempts were still carried out by the scientists in this field to visualize and track biomolecules with CNTs.

In 2006, Lin et al. [116] developed a sensitive approach to detect the translocation of the lipid-single wall CNT (SWNT) assembly in cells via energy transfer. Earlier research showed that as the fluorophores directly attached to the surface of CNTs, both energy-transfer and electrontransfer processes would induce nonradiative relaxation for excited fluorophores on CNT surfaces [117]. In order to prevent this quenching process, some molecules such as polyethylene glycol (PEG), porphyrin and its derivatives have often been applied to connect fluorophores and CNTs $[118,119]$. For instance, Jia et al. [119] reported in vivo and in vitro fluorescent imaging studies on porphyrin-CNTs conjugates in 2009. During their experiments, they found that the unique fluorescent properties of this conjugate enable a detailed tracking of transportation process of these conjugates as they were injected into mice.

There are some other CNT-related fluorescent applications, such as CNT-quenched fluorescent oligonucleotides and noncovalent assembly of CNT and single-stranded DNA $[120,121]$. The relevant literature base for CNTs has fewer publications than that of QDs; nevertheless, these demonstrations already show promise in biological fluorescence 
detection. However, there are some obstacles that limit the applications of CNTs into living cells and are also some basic issues which hinder the usage of CNTs for other applications. For example, it is still difficult to precisely control the chirality of CNTs experimentally. Consequently, it is quite challenging to separate large quantities of strongly fluorescent SWNTs. Therefore, impurities inside the samples such as metallic nanotubes actually inhibit the brightness of their fluorescent semiconductor nanotube neighbors. Fluorescence properties of semiconductor nanotubes themselves also have varying degrees of fluorescence efficiency since approaches to choose CNTs with the same properties have not been refined. In addition, strong intermolecular interactions between CNTs usually create CNT bundles and although alternatives have been proposed to decrease CNT aggregation, including the use of extreme ultrasound to break apart bundles of nanotubes and force them to dissolve, these techniques can cause defects thus decrease CNT effectiveness. Research on CNTs has shifted in the more recent years towards graphene, where its first published report in 2004 provided an alternative to struggles with creating usable CNT systems [21].

Recently, graphene, a free-standing 2D carbon film with single-atom thickness, has become a highly studied material in the field of science. The large planar structure of graphene makes it an ideal substrate for adsorption of different kinds of biomolecules. Up to now, various graphene-based nanostructures have been utilized to fabricate functionalized bio-systems integrated with various biomolecules, such as nucleic acids, peptides, proteins and even entire cells. Conversely, graphene-based fluorescence bio-detection has been demonstrated to be a successful technique due to graphene's superior quenching efficiency towards biomolecules. The ability of graphene to quench electron donors from biomolecules absorbed on the graphene such as single-stranded DNA (ssDNA), has allowed for graphene to be developed into a rapid, rigid, highly sensitive and highly selective FRET biosensor.

The first graphene-based FRET biosensor was developed by Lu et al. [122] in 2009 where they demonstrated the ability of graphene oxide to provide a platform for the detection of DNA and proteins. In order to absorb DNA and proteins, water soluble GO was used. Usually, for FRET biosensors, an energy transfer process from a donor fluorophore to an acceptor fluorophore is involved for this kind of sensor. $\mathrm{Lu}$ et al. [122] found that the fluorescence signal rapidly quenched as the dye-labeled ssDNA adsorbed onto GO. The addition of the complementary DNA strand altered the conformation of the dye-labeled DNA and caused its release from $\mathrm{GO}$ and the eventual restoration of fluorescence signal. The results of the above experiment show the versatility of GO-based FRET sensors and can be extended, by different ssDNA sequences, to include multicolor DNA analysis [123]. Similarly, a graphene-based FRET has been developed as an assay for DNA helicase unwinding that depends on the preferential binding of GO to ssDNA over dsDNA, thereby inducing quenching of fluorescent dyes conjugated to ssDNA [124]. Specifically, a GO-dsDNA substrate containing a fluorescent dye at the end of one strand was prepared first, and then DNA helicase was injected into the system which initiated the helicase reaction that led to intense fluorescence of dsDNA. As the helicase-induced unwinding of dsDNA proceeded, the fluorescence decreased rapidly due to strong interactions of GO with unwound ssDNA, thus enabling the monitoring of the helicase activity in real time by following the change in fluorescence [124].

Besides, in order to improve the sequence-specific detection of target-DNA, molecular beacons (MBs), which are dually labeled single-stranded oligonucleotide hybridization and form stem-and-loop structures to bring a fluorophore and a quencher together and result in fluorescence quenching of the fluorophore, have been used to fabricate graphenebased FRET bio-analytical sensing where MBs with an "on/off" switching design would be highly desirable [125]. Since the onset of MB development [126], they have been used for widespread applications ranging from molecular and cellular biology, to pathogen detection and biomedical diagnostics $[127,128]$.

In spite of their extensive capabilities, they are in most ways limited due to insufficient sensitivity, difficulty in their synthesis, and selection of suitable FRET pairs in some certain cases [120,129]. Nevertheless, when graphene is combined with MBs in FRET bio-detection, graphene can act as a nanoquencher for fluorophores and can also serve as a nanoscaffold for MBs. That is, a much easier synthesis of biosensors as well as higher sensitivity would be obtained at the same time on MB-graphene-based FRET biosensors. Recently, many groups reported their success in fabricating such FRET biosensors to probe various biomolecules, such as DNA, thrombin, some metal ions, small molecules, and proteins $[123,130,131]$. Figure 5 gives a brief illustration of various biomolecules attached to graphene-MB-based FRET biosensors.

\section{Surface plasmon resonance}

With the help of labeling and probing, the above-reviewed fluorescence technique is a powerful imaging method to study the nano-bio systems. Another versatile technique for nanomaterial-based biosensing and optical sensing is surface plasma resonance (SPR), whose advantages include real-time detection, high sensitivity, label-free detection, and quantitative sensing capacity [133]. In the following section, we will introduce the applications of nanomaterials to SPR- based bio-detection, the sensitivity improvement by localized surface plasmon resonance (LSPR), and the fabrication of metallic nanoparticle arrays to accelerate the development of nanomaterial-based LSPR biosensors. 


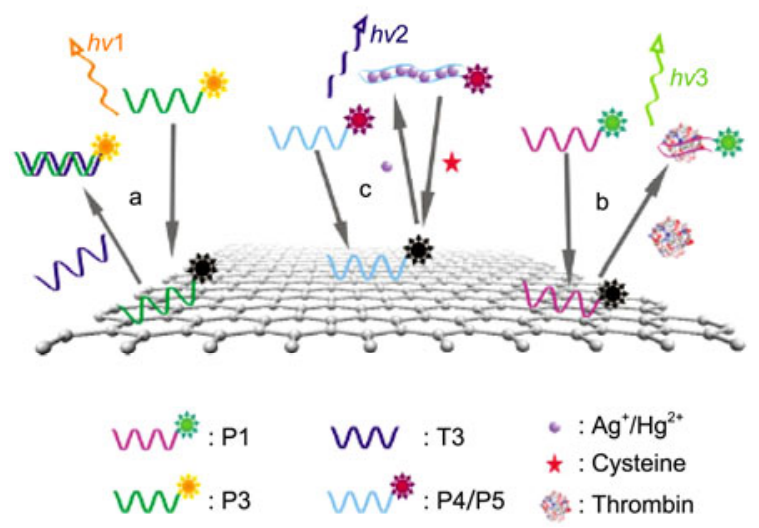

Figure 5 Schematic illustration of the ssDNA-GO architecture platform for multiplex targets detection. (a) DNA, (b) thrombin, (c) $\mathrm{Ag}^{+}, \mathrm{Hg}^{+}$, and cysteine. Reproduced from Ref. [132] with permission from Elsevier.

\subsection{Application of nanomaterials to SPR-based bio-detection}

SPR was first developed by Liedberg et al. [44] in 1983 and is the most widely applicable tool for measuring adsorption of material onto planar or nanoparticle metal surfaces. It can generally be described as the resonant, collective oscillation of valence electrons in a solid stimulated by incident light. For planar SPR, p-polarized light is coupled into a thin metal film by total internal reflection through a prism, also known as the Kretschmann conformation [134]. A resonance condition is established when the frequency of these photons matches the resonant oscillation frequency of the surface plasmon polaritons (SPPs). This resonance condition is sensitive to macroscopic properties of these surfaces including index of refraction, therefore small changes in the index of refraction of the metal cause detectible SPR signal. This technology has found substantial use in biomolecule adsorption measurements.
By taking advantage of developments in nano-fabrication and nanoparticle synthesis technologies, designs of an optical biosensor using nanostructured metals to produce localized surface plasmon resonance (LSPR) has experienced great development through the past decade. Compared to SPR, LSPR can be excited in nanostructured metal by light incident at any angle and it is thought that this incident light induces the conduction electrons in the metal to oscillate collectively [135]. The peak position and intensity of LSPR highly depends on nanoparticle composition, size, shape, spacing, and surrounding environment [47,136-139]. Generally, white light is used as the excitation source for LSPR and when the wavelength of light matches the frequency of localized SPPs, the phenomenon shows that the light has been absorbed and scattered by the sample. LSPR spectra measure the light extinction and scattering of the nanoparticles.

\subsection{Sensitivity improvement of LSPR}

As stated in the previous section, the LSPR extinction wavelength maximum $\lambda_{\max }$ is related to refractive index. Changes in refractive index in local environment, such as a protein binding event, shift the observed $\lambda_{\max }$ in frequency. We can relate the spectral shift $\lambda_{\max }$ and local refractive index change $\Delta n$ by $[135,139,140]$ :

$$
\Delta \lambda_{\max }=m \Delta n\left[1-\mathrm{e}^{\left(\frac{-2 d}{l_{\mathrm{d}}}\right)}\right],
$$

where $m$ is the bulk refractive-index response of the nanoparticles (in nanometers per refractive index unit (RIU)), $\Delta n$ is the change in refractive index induced by adsorbate, $d$ is the effective thickness of adsorbate layer (in $\mathrm{nm}$ ), and $l_{\mathrm{d}}$ is the electromagnetic field decay length (in nm). Figure 6
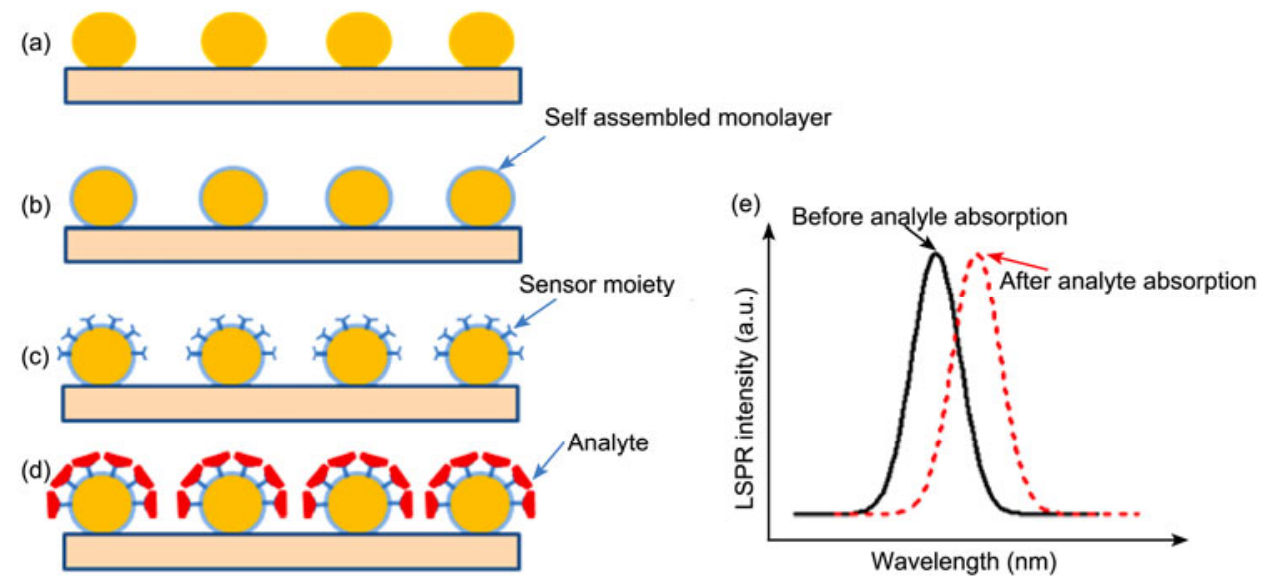

Figure 6 Schematics of LSPR sensor. (a) Metal nanoparticles are coated on a glass substrate; (b) silver nanoparticles coated with SAMs; (c) metal nanoparticles are modified as a sensor moiety with an analyte recognition function; (d) analyte adsorbed onto the nanoparticles through the specific binding of targed molecules; (e) LSPR shift in response to the analyte adsorption. 
demonstrates how an LSPR sensor works. Typically in LSPR sensors, nanoparticle arrays are deposited or chemically bound on the solid support and act as a sensor transducer (Figure 6(a)). Usually, these metallic nanoparticles have been coated with a self-assembled-monolayer (SAM) which stabilizes and functionalizes the NPs (Figure 6(b)), providing a reactive end group to enable the sensor moiety (Figure 6(c)). Some special SAMs containing ethylene glycol (EG) are often chosen because they also can prevent non-specific adsorption. Through measuring an LSPR shift shown in figure 6e in response to analyte adsorption (figure 6(d)), it is possible to quantify the amount of adsorbed analytes onto these functionalized NPs. LSPR biosensing is focused in the pursuit of high sensitivity which is very important for plasmonic sensors to detect low concentrations of analyte or small molecules in solution.

Sensitivity can be increased in two aspects in LSPR; either by increasing LSPR signal while narrowing its bandwidth or enhance LSPR shift. Higher intensity and narrower LSPR band can improve both the signal-to-noise ratio and spectral resolution allowing for calculating $\lambda_{\max }$ in real time. This increase in signal makes LSPR a powerful tool in measuring real-time binding kinetics [136]. From eq. (1), we know that the refractive index change is directly proportional to the shift in $\lambda_{\max }$. Anker and co-workers [141-143] discussed several ways to increase LSPR shift [136]: (1) larger molecules produce a greater change in the local index of refraction, (2) chromophores that absorb visible light couple strongly with the LSPR nanoparticles which lead to a substantial shift in resonance frequency [141-143], (3) nanoparticles who are spaced less than about 2.5 radii show strong plasmonic coupling which can lead to increased signal.

The concentrations of small biomolecules such as hormones, neurotransmitters, and pheromones in the blood stream or saliva can often be indicative of the bio-function of an organism. Sensing of these small molecules in complex matrices poses a challenge because of high mass mol- ecules such as proteins interfering with target molecules in small quantities [144]. Using labeled nanoparticles increases the local dielectric constant and also can introduce plasmonic coupling (as stated above) resulting in higher sensitivity to smaller target biomolecules [144-151]. As shown in Figure 7, the silver nanoparticle-based LSPR biotin biosensor responds with a very large change upon attachment of biotin-labeled gold nanoparticles. The increased mass due to the attached nanoparticle, plus the resonance enhancement between gold nanoparticles lead to a 4-fold increase in LSPR signal. The addition of the gold nanoparticles leads to three orders of magnitude improvement over traditional SPR measurements. Figure 8 shows the coupling between metal NPs leading to the LSPR shifts. With decreasing distance between nanoparticles, the LSPR spectra red shift exponentially due to an increase in scattering intensity [152].

Small biomolecules are a difficult challenge for SPR detection since they do not lead to significant changes in the local index of refraction. In order to detect such analytes, larger protein receptors such as antibodies have been utilized. Lee et al. [153] measured amplified LSPR signal by employing enzyme-based attachment. In their work, the intermolecular binding between an enzyme and antibody target fixed on a gold nano-island (NI) resulted in a two order of magnitude increase in their detection limit.

Unlike SPR, LSPR decay length is much shorter and on the same size scale as proteins. Hence, the conformation changes of proteins at the surface may cause large spectral change in the LSPR signal collected [154]. Hall et al. [155] were the first to demonstrate conformational changes of the unlabeled protein calmodulin $(\mathrm{CaM})$ with the modulation of $\mathrm{Ca}^{2+}$ using LSPR. In the presence or absence of calcium ions, CaM adopts two different conformational states and switches from "open" to "close" state upon calcium binding. This causes the center-of-mass of CaM to localize out or into the LSPR sensing region. The response of $2 \mathrm{mmol} / \mathrm{L}$
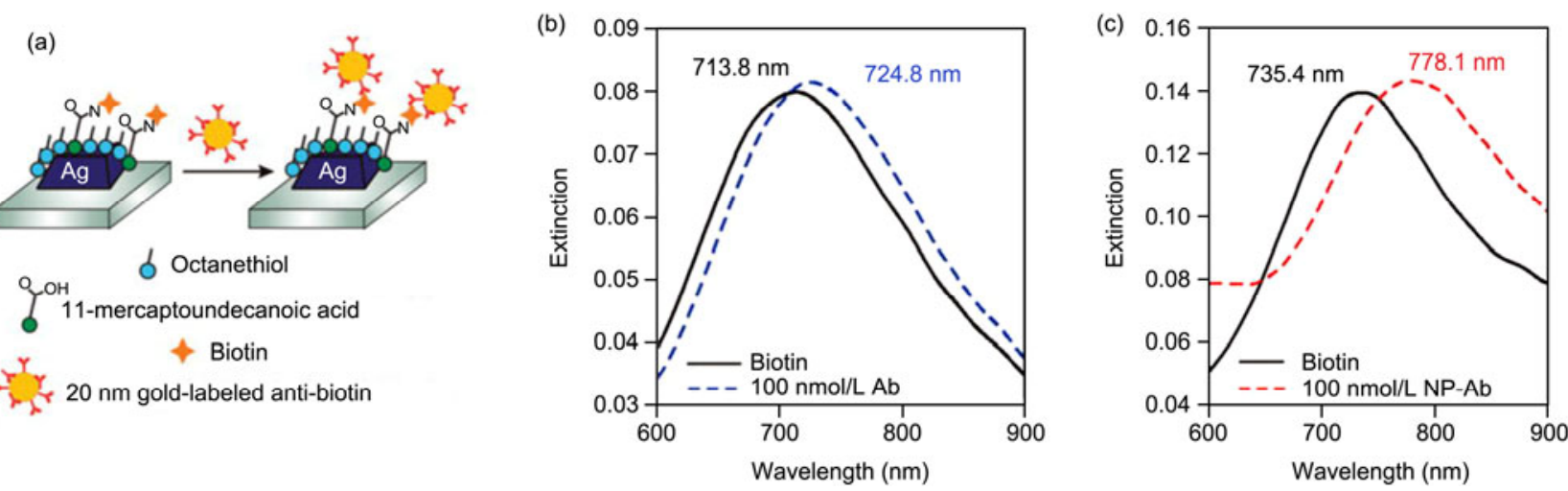

Figure 7 Illustration of increasing of LSPR sensitivity by using labeled nanoparitcles. (a) Biotin is covalently linked to a nanoparticle surface using EDC coupling agent, and antibiotin labeled gold nanoparticles are subsequently binding to the surface. (b) LSPR spectra before (solid black) and after (dashed blue) binding of native antibiotin, showing a shift of $11 \mathrm{~nm}$. (c) LSPR spectra before (solid black) and after (dashed red) binding of antibiotin labeled nanoparticles, showing a shift of $42.7 \mathrm{~nm}$. Reprinted with permission from [151]. Copyright 2011 American Chemical Society. 

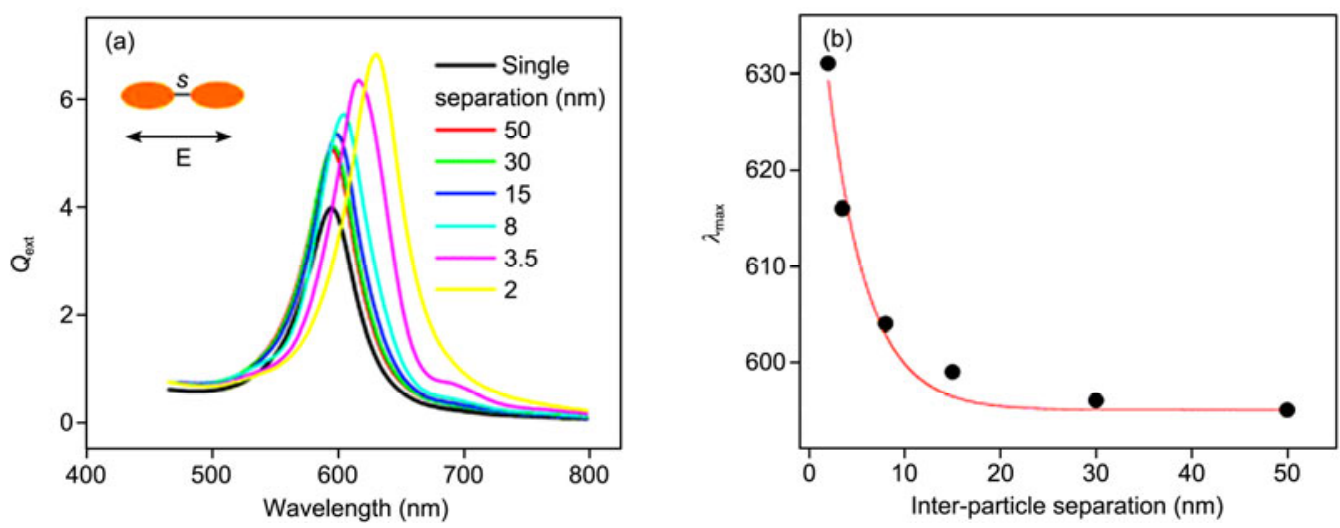

Figure 8 (a) LSPR spectra of a pair of gold spheroids as a function of the interparticle separation. The spectrum of a single gold spheroid (short axis= $10 \mathrm{~nm}$, long axis $=20 \mathrm{~nm})$ is shown in black. (b) Plasmon resonance wavelength red shifts exponentially $\left(R^{2}=0.98063\right)$ with decreasing interparticle separation in the spheroid pair. The fitting curve offset, $595 \mathrm{~nm}$, compensates for the single-particle resonance wavelength. Reprinted with permission from [152]. Copyright 2008 American Chemical Society.

$\mathrm{Ca}^{2+}$ results in a resonance frequency shift of $2.2 \mathrm{~nm}$, which is far greater than the noise level.

Recently, several groups have been trying to design nanoparticle array structures using nano-patterned substrates. Fujieda et al. [156] attempted to use CNTs as a support to organize metal particles in three dimensions, improving overall sensitivity. Substantial work has been done to demonstrate that this proposed substrate has similar functionality as an LSPR sensor. Zou et al. [157] reported a synthesis of novel nanostructures, in which silver clusters were embedded in porous silica, for the detection of nitro explosives. Porous silica adsorbing polar nitro explosives leads to an enrichment of nitro explosives molecules around the embedded silver clusters and hence strongly increasing the detection of these target materials, with a detection limit of less than $1 \mu \mathrm{mol} / \mathrm{L}$.

\subsection{Fabrication of metallic nanoparticle arrays}

The peak center of LSPR spectra highly depends on nanoparticle composition, size, shape, and spacing. Gold and silver are the mostly used materials for LSPR nanoparticles. Compared to gold, silver nanoparticles have a smaller resonance bandwidth and a higher sensitivity [157]; however it is more likely to oxidize and has a higher biological toxicity [154]. In the previous section, we introduced that the distance between metallic nanoparticles has a profound effect on LSPR spectra in both intensity and spectral shifts. Size also plays an important role in shape and position of LSPR spectra. The diameter of spherical gold nanoparticles has a resonance bandwidth from 9-99 $\mathrm{nm}$, which generates a $\lambda_{\max }$ from 520 to $580 \mathrm{~nm}$. Larger sphere nanoparticles have bigger value, but suffer from a lower resolution. For shape effect: Experimental and theoretical research has been done on determining relationships of nanoparticle shape. Results have shown that sharper nanoparticles give rise to higher sensitivity to local refractive index [136]. The spectra of silver triangular and cube nanoparticles are more sensitive to external refractive index than spherical silver nanoparticles. In general, increasing the ratio of width/height of nanoparticles can cause the redshifts in LSPR spectra, higher $\mathrm{m}$ value and a longer evanescent field length [136], which can directly relate to an enhancement in LSPR sensors. Figure 9 shows that SPR sensitivity increases with decreasing

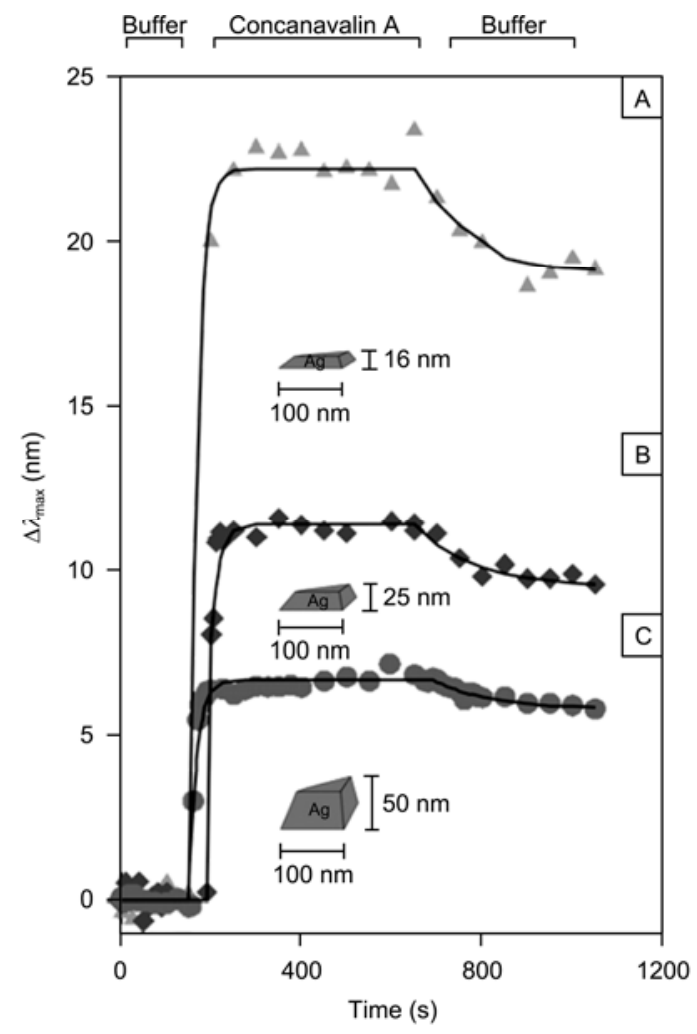

Figure 9 Time dependent curves of mannose-functionalized silver nanosensors in response to $19-\mu \mathrm{mol}$ concanavalin A for different out-ofplane heights. Reprinted with permission from [158]. Copyright 2004 American Chemical Society. 
distance away from the metal surface. This was demonstrated for triangular prism-shaped silver nanoparticles with identical base dimensions and decreasing heights [158].

Uniform metallic nanostructure array fabrication has greatly impacted LSPR sensor development. The uniformity of nanoparticle size, shape or spacing determines LSPR resolution, and sample reproducibility. Currently, the most common method for nanoparticle array fabrication is nanosphere lithography (NSL); known for being cost-effective, rapid, and easy to control. Figure 10 illustrates the NSL process: (1) A mask of polymer nanospheres are first deposited on the surface of a substrate, (2) the nanospheres, acting as mask, shape the design for the deposited metal (typically 15-100 nm), (3) and after deposition, the polymer nanospheres can be washed away. This process generates a triangular nanoparticles array as shown in Figure 10 [15]. If a thicker metal layer is deposited over the nanospheres, a thin metal film over nanosphere (FON) can be fabricated. Varying the diameter of nanospheres and thickness of deposited metal allows for tunability in the resonance wavelength of these LSPR sensors. In addition to NSL, nanoparticle array fabrication techniques such as electron beam lithography, focused ion beam lithography, and nano-imprint lithography have made great progress. These techniques have provided greater controls on size, shape, and spacing of nanoparticles arrays, which can further accelerate the development of nanomaterial-based LSPR biosensors.

\section{SFG vibrational spectroscopy}

With the density and availability of surface area in many biological systems, most nanoscale interactions can be thought of as interactions with a surface. Likewise, it is of much importance to understand and characterize these surface interactions. There are several surface detection methods such as surface plasmon resonance (SPR), fluorescence and electron microscopic techniques, atomic force microscopy (AFM), X-ray photoelectron spectroscopy (XPS), attenuated total reflectance Fourier transform infrared (ATRFTIR), Fourier transform infrared reflection absorption

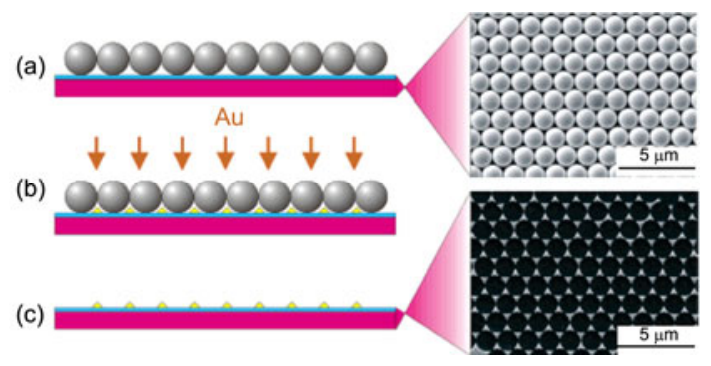

Figure 10 (Color online) Illustration of the process of nanosphere lithography (NSL). (a) Deposition of a mask of polystyrene nanospheres on a substrate; (b) depositon of metal (Au) on nanosheres by thermol evaporation; (c) removal of the nanosphere mask. Reprinted with permission from [15]. Copyright 2005 American Chemical Society. spectroscopy (FT-IRRAS), and surface-enhanced Raman spectroscopy (SERS). However, these techniques cannot simultaneously fulfill the need to selectively monitor the molecular details of the surface and interface and avoid the interference of the information from species in the bulk. Specifically, although FT-IRRAS and SERS have the capability to probe surface chemistry with molecular resolution, FT-IRRAS generally requires a reference spectrum in order to sort out the interfacial signal and SERS needs specially prepared metal surfaces or the use of nanoparticles of the order of several nanometers to $100 \mathrm{~nm}$ for electromagnetic and/or chemical enhancement [159]. One surface-specific technique that has been proven to yield molecular level structure information (e.g. on both the orientation and degree of ordering of adsorbed chemical groups) is sumfrequency generation vibrational spectroscopy (SFG).

We will first introduce the principle of SFG and its applications in biological surface and interface systems. After that, recent advancements that explore the utility of SFG to the nanoparticle-based systems are summarized.

\subsection{SFG: A tool for the biological surface and interface}

SFG is a second order non-linear optical technique where two pulsed laser beams, a fixed frequency visible beam and a frequency tunable infrared beam, are overlapped onto the sample surface. This gives rise to a nonlinear optical response which generates a third beam at a frequency that is the sum of the frequencies of the two incident beams (Figure 11) [52,160-165]. The intensity of the output light (SFG signal) is proportional to the second-order non-linear susceptibility which equals zero when a material has inversion symmetry under the electric dipole approximation. The majority of bulk materials possesses inversion symmetry and therefore do not generate sum frequency signal, making SFG a truly surface and interface sensitive technique. For chemical groups at a surface or interface, SFG signal is generated when coincides with the energy gap of their vibrational modes. This resonance frequency enhancement is unique to each chemical group and can therefore provide the information of surface chemical composition. Alignment,

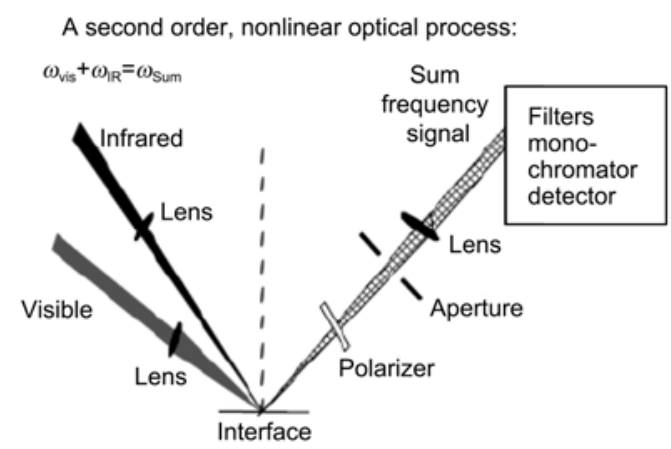

Figure 11 SFG experimental geometry. Reproduced from Ref. [52] with permission from Annual Review. 
orientation, and orientation distribution (or ordering) of surface functional groups can be deduced by examining SFG spectra collected using different polarization combinations of input and output beams [52,160-165].

Since the first SFG paper published in 1987 [166,167], SFG has been widely used to investigate many different surfaces and interfaces in situ in real time. It has been demonstrated that SFG vibrational spectroscopy is capable of probing interfacial structures of a variety of polymer and biological surfaces and interfaces [53-55,168].

SFG has been applied to study proteins and peptides and their interactions and orientations at various interfaces [58,169-185]. In the recent years, methods have been developed to determine conformation and orientation of various protein secondary structures at interfaces using polarized SFG, supplemented by ATR-FTIR spectroscopy [57, $61,66,68,70,80,186,187]$. Molecular level understanding on peptide and protein structures at interfaces using SFG provides a foundation to understand molecular mechanisms of biocompatibility, marine biofouling, membrane protein functions, biosensing, and antimicrobial peptide activity and selectivity. It has been demonstrated that SFG is a unique technique to determine membrane orientation of various $\alpha$-helical peptides such as magainin-2 [69], MSI-78 [74], and melittin [68]. A cell penetrating peptide, Pep-1, which can form both $\alpha$-helical and $\beta$-sheet was also studied [56]. SFG has also been applied to study orientations of larger membrane proteins with $\alpha$-helical structures such as cytochrome b5 [71], G $\beta \gamma$ [67], and G $\beta \gamma$-GRK2 complex [75]. The protein complex formation at model cell membrane surface has been observed in situ in real time using SFG. In addition to the $\alpha$-helical peptides, methods to deduce membrane orientations of a $3_{10}$ helical structure and $\beta$-sheet structure using SFG have also been developed and applied to examine membrane orientation of alamethicin [64,188] and tachyplesin I $[76,80,186]$. SFG is also used to study the formation of $\beta$-sheet aggregates from human islet amyloid polypeptide (hIAPP) on membrane surfaces [175]. Besides membrane peptides and proteins, SFG has also been used to investigate physically adsorbed and chemically immobilized peptides and proteins at liquid/air [181] or solid/liquid interfaces [57,62,63,65,189-192].

The interactions of large proteins, DNA, and biofunctional synthetic polymers with cellular membranes have great importance not only for their size similarities to common nanomaterials discussed above, but also their unique structure/function relations with their interfacial environment. Shown in Figure 12 is a recent advancement in the application of SFG towards the investigation of the interaction between cell membrane and the complex formed by $\mathrm{G}$ protein-coupled receptor kinase 2 (GRK2) and G $\beta \gamma$ subunit [75]. It was found that the likely membrane orientation of the GRK2-G $\beta \gamma$ complex differs from that predicted from the known protein crystal structure. Instead, a more likely picture holds the predicted receptor docking site of GRK2 (a)
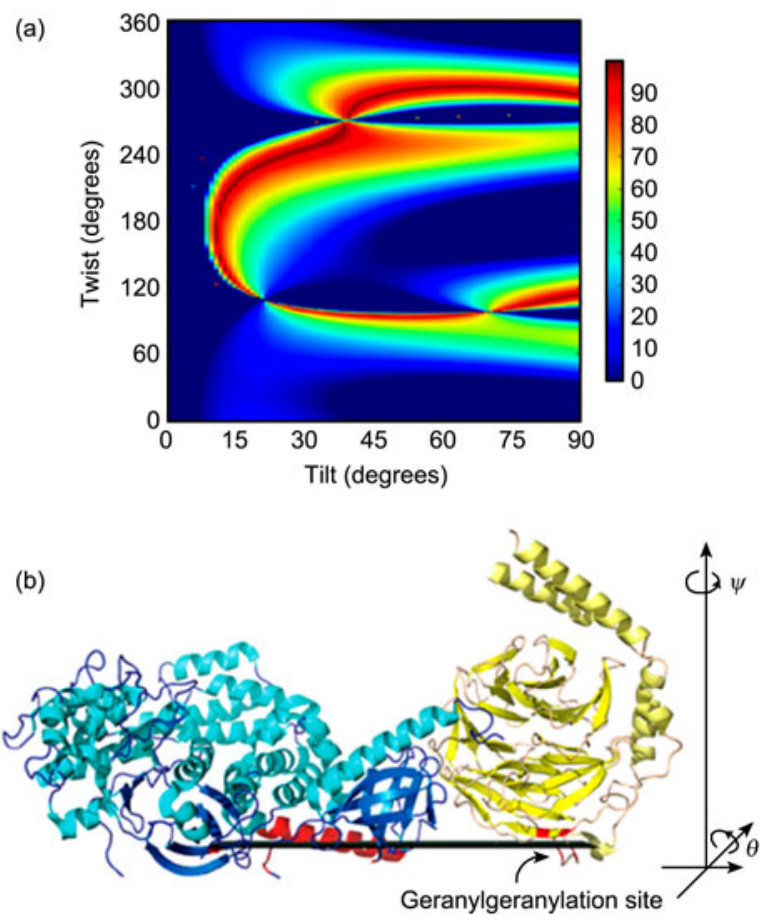

Figure 12 (a) Possible orientation of the G $\beta \gamma$-GRK2 complex determined by polarized SFG measurements and the use of the computer software; (b) complex at the $(0,0)$ orientation [75].

in an orientation that would more optimally interact with GPCRs. It was also found that G $\beta \gamma$ appears to change its orientation after binding to GRK2. This research demonstrates that SFG can be used to observe the protein complex formation in situ in real time, and can be used to deduce membrane orientation of large protein complexes.

\subsection{SFG studies at the nano-bio interface}

Up to now, despite its applicability to various systems, SFG spectroscopy has been mainly applied to planar geometries. Recent studies have demonstrated that SFG is a powerful and information-rich probe of nanostructured surfaces [193-195], and surface behaviors with nanoparticles [159,196-200]. In the following sections, recent advancements that explore the utility of SFG as a powerful and unique technique to study surfaces/interfaces containing nanoparticles will be covered. Structures comprising metallic or semiconductor nanoparticles have potential applications in a diverse range of technologically significant fields. Nanoparticles can exhibit various electrical, optical, magnetic, chemical, and biological properties by tuning parameters such as their composition, size, and shape, having versatility as a functional material in the fields of magnetic storage media, biosensor, and medication. Many of the optical, transport, catalytic, and thermodynamic properties of these nanoparticles are surface related, and the properties of these nanoparticles after modification by adsorbing surfactants and organic molecules are therefore of great interest. Conformation of adsorbed mole- 
cules often determines the physical, chemical, and biological properties.

SFG can be used to characterize the structure of nanoparticle surfaces. The most widely used nanoparticles are gold nanoparticles (AuNPs) which have the following unique properties: (1) they are relatively inert, (2) they can be easily functionalized through thiolate groups, (3) the synthesis of size controlled AuNPs is quite easy, and (4) their plasmon characteristics make them ideal candidates for biolabeling. Small AuNPs are usually covered by a layer of stabilizing agent (charged small molecules or surfactants). Studying the physicochemical properties of these adsorbed molecules at the surface of nanoparticles is very important to understand the fundamental problems toward their applications. A recent example was studied by Benderskii and co-workers who used SFG to study the conformation of dodecanethiol covalently linked on AuNPs with four different sizes: 1.8, 2.9, 7.4, and $23 \mathrm{~nm}$ [193]. The SFG spectra (SSP polarization; where each element is the polarization of the signal, IR, and visible beams, respectively) recorded in the $\mathrm{CH}$ stretch region are shown in Figure 13. The observed vibration modes can be assigned as $\mathrm{CH}_{2}$ symmetric stretch $\left(\mathrm{d}^{+}\right)$at $2855 \mathrm{~cm}^{-1}, \mathrm{CH}_{2}$ antisymmetric stretch $\left(\mathrm{d}^{-}\right)$at $2918 \mathrm{~cm}^{-1}$, $\mathrm{CH}_{3}$ symmetric stretch $\left(\mathrm{r}^{+}\right)$at $2881 \mathrm{~cm}^{-1}$, and $\mathrm{CH}_{3}$ asymmetric out-of-plane stretch at $2950 \mathrm{~cm}^{-1}$. A weaker band at $2935 \mathrm{~cm}^{-1}$ may be assigned to the Fermi resonance between $\mathrm{CH}_{3}$ symmetric stretch and bend overtone modes. The

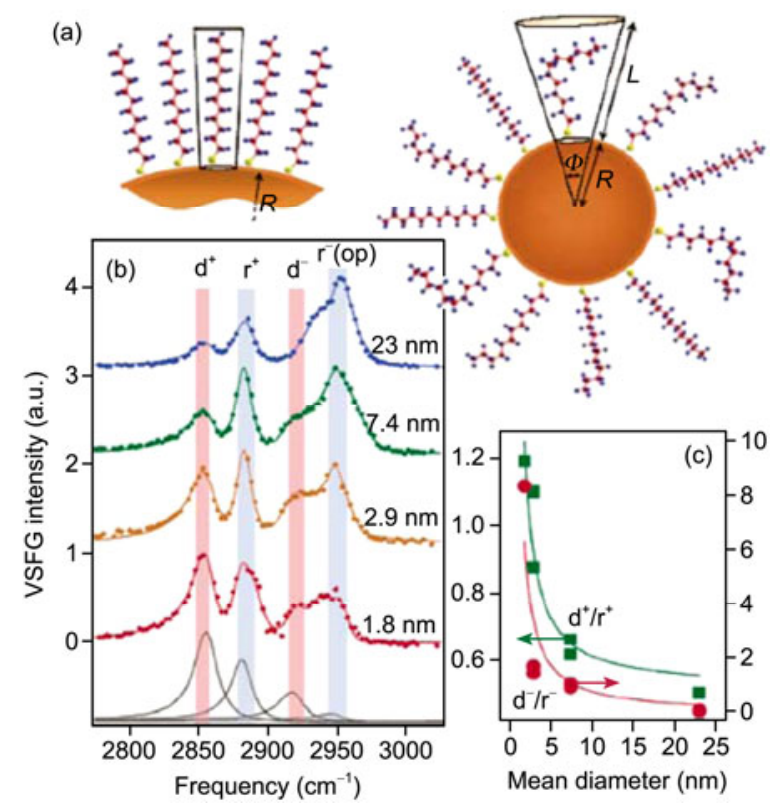

Figure 13 (Color online) (a) Schematic representation of the dodecanethiol ligand on spherical nanoparticle surfaces showing conical volume available for the formation of gauche defects. (b) Size-dependent SFG spectra (SSP) of the dodecanethiol on gold nanoparticles in the $\mathrm{CH}$ stretch region. (c) The ratio of the methylene $\left(\mathrm{d}^{+}, \mathrm{d}^{-}\right)$to methyl $\left(\mathrm{r}^{+}, \mathrm{r}^{-}\right)$mode intensities in the SFG spectra showing conformational change (increase of gauche defects) as a function of particle size. Reprinted with permission from [193]. Copyright 2006 American Chemical Society. authors noted that the relative intensity increase of the $\mathrm{CH}_{2}$ versus $\mathrm{CH}_{3}$ transitions was inversely proportional to the particle size. This is particularly obvious for the symmetric stretches, $\mathrm{d}^{+}$versus $\mathrm{r}^{+}$, which are well separated from other modes (Figure 13(b)). If the alkyl chains are predominantly in all-trans conformation, the SFG signal intensity of methylene modes is very weak due to the local symmetry of the $\mathrm{CH}_{2}$ groups. The intensity of the methylene modes will increase as the content of gauche conformation increases. Thus, the relative intensity of the $\mathrm{d}^{+}$and $\mathrm{d}^{-} \mathrm{CH}_{2}$ modes with respect to the $\mathrm{CH}_{3}$ modes (Figure 13(c)) can be viewed as a semi-quantitative measure of the extent of gauche defects in the ligand alkyl chains. The nanoparticle size-dependent results clearly show that the content of gauche defects is higher in smaller-sized nanoparticles (Figure 13(a)). This is a typical example showing the dependence of molecular conformation on the geometry of the nanoscale substrate.

Another successful attempt of SFG characterization was achieved by Davies and co-workers on a cationic surfactant deposited on anionic stabilized AuNPs forming a monolayer on a silicon substrate [195]. By recording SFG spectra $(\mathrm{CH}$ stretch region) of both a dioctadecyldimethylammonium chloride (DODAC) monolayer deposited on a silicon substrate, and a composite gold nanoparticle/DODAC film deposited on a silicon substrate, they showed that the latter spectrum is significantly different from the former. To explain this, they proposed two possibilities: (1) the difference may be due to differences in the visible and infrared electromagnetic fields generated at the surfaces when using nanoparticle rather than silicon substrates. The electromagnetic field strengths affect not only the magnitude of the background sum frequency signal but also the relative importance of the individual tensor components of the resonant second-order nonlinear susceptibilities and hence the strengths of each resonance. Different consequences of changes in these fields between silicon, gold nanoparticles, and evaporated gold substrates can be expected. Silicon is a poor reflector in the infrared and consequently the surface electric field established perpendicular to the surface ( $z$-axis) is small. In contrast, the infrared reflectivity of gold is high and increases to nearly $100 \%$ at thicknesses of the order of hundreds of nanometers. If the nanoparticle film is considered to be a very thin gold layer on the silicon substrate it follows that the surface infrared electric field produced will have a larger $z$ component than would be present on bare silicon, but smaller than that which would exist on thick gold. The absolute and relative intensity of individual resonances would therefore be expected to depend on the degree of interaction with the infrared field in the $z$ axis. (2) Regarding the interaction between laser and nanoparticle, it is also important to discuss the surface plasmon resonance effect. Silicon has negligible surface plasmon resonance and no enhancement of the surface E-fields is expected by this mechanism. In contrast, bulk gold exhibits strong wavelength independent surface plasmon resonance which results 
in enhancement of the $z$-axis surface E-field. Differently, the surface plasmon resonance of gold nanoparticles is wavelength specific having a maximum at $\lambda \approx 530 \mathrm{~nm}$ for a $15 \mathrm{~nm}$ diameter nanoparticle, which coincides with the wavelength of visible light. Consequently, the visible surface E-field will have an enhanced intensity in comparison to silicon and like all surface plasmon fields, will contain only a $z$ component. Preferential enhancement of resonances with significant $z$ components would therefore be expected.

Besides SFG studies on the surface of nanoparticles, another related aspect is focused on the surface behaviors containing nanoparticles. The first attempt to explore the utility of SFG to the field of surface catalysis was achieved by Somorjai [201] in 1997. Ordered arrays of Pt nanoparticles in the $2.5-50 \mathrm{~nm}$ size range were deposited on oxide substrates (silica, alumina, and titania), which were used in catalyzed surface reactions at high pressures. SFG was used to monitor pressure-dependent changes in the chemisorption of $\mathrm{CO}$ and $\mathrm{NO}$ over $\mathrm{Pt}(111)$. The bonding process and the formation of reaction intermediates were detected by SFG. Later, Rupprechter used SFG to study the CO molecules adsorbed on $\mathrm{Pd}$ or Pt nanoparticles at controlled pressure and temperature [202,203]. Yeganeh et al. [197] demonstrated that the total internal reflection (TIR) configuration reduces destructive interference associated with non-linear optical spectroscopy of small particles making SFG studies of small objects possible. In a similar way, SFG spectroscopy in total internal reflection geometry was successfully applied to study the adsorption and oxidation of $\mathrm{CO}$ on monolayer films of platinum cubic nanoparticles [198]. These examples demonstrate the versatility and strength of SFG to obtain the molecular level detection of reaction intermediates and the dynamic restructuring of surfaces during catalytic reactions.

Davies and co-workers [199] used SFG to study the nanoparticle-induced structural changes within fatty acid multilayer films. They prepared films from a total of nine layers of per-deuterated cadmium arachidate and one layer of perprotonated cadmium arachidate on hydrophobic per-deuterated octadecanethiol (d-ODT) covered gold substrates. Four samples were prepared containing a single per-protonated cadmium arachidate layer, namely layers $1,2,5$, and 10 , respectively, counting from the lowermost layer to the surface layer. They collect SFG spectra in the $\mathrm{CH}$ stretching region before and after reaction with $\mathrm{H}_{2} \mathrm{~S}$ (the addition of the $\mathrm{H}_{2} \mathrm{~S}$ gas can induce the formation of $\mathrm{CdS}$ nanoparticles in the interlayer regions of these multi-layers in situ). The SFG spectra of the unreacted films are shown in the left panel of Figure 14. The most striking feature is that the resonances of layers 2 and 10 occur as dips, while those of layers 1 and 5 occur as peaks. This is due to interference between the SFG signals from the gold substrate and the SFG active (per-protonated) arachidate layer and indicates that the methyl groups of layers 2 and 10 point away from the surface while the methyl groups of layers 1 and 5 point

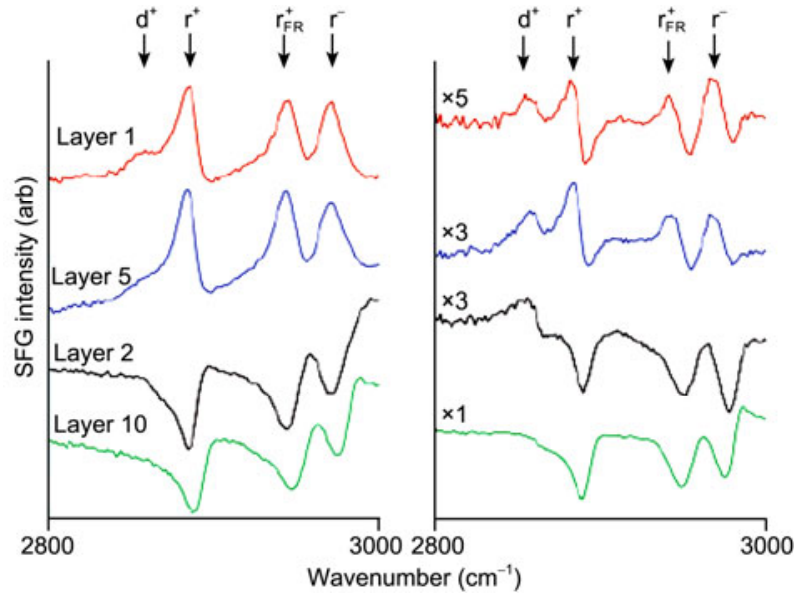

Figure 14 SFG spectra of a single per-protonated cadmium arachidate layer in an otherwise fully per-deuterated 10-layer film deposited onto d-ODT gold before (left) and after (right) reaction with $\mathrm{H}_{2} \mathrm{~S}$. The spectra correspond from top to bottom to layers $1,5,2$, and 10 , respectively. The symbols refer to the symmetric methylene stretching mode $\left(\mathrm{d}^{+}\right)$, the symmetric methyl stretching mode $\left(\mathrm{r}^{+}\right)$, its Fermi resonance $\left(\mathrm{r}^{+} \mathrm{FR}\right)$, and the asymmetric stretching mode $\left(\mathrm{r}^{-}\right)$. Post-reaction spectra have been rescaled as indicated by the multiplication factors. The nonresonant background remained the same post-reaction. Reprinted with permission from [199]. Copyright 2004 American Chemical Society.

toward the surface. The three strong resonances arise from methyl groups, while a weak methylene resonance around $2860 \mathrm{~cm}^{-1}$ can be observed for layer 1 . This implies that the hydrocarbon chains are in an all-trans configuration, but some gauche defects do occur for layer 1. Figure 14 (righthand panel) shows the SFG spectra of layers 1, 5, 2, and 10, respectively, after reaction. It can be seen that the intensity of the SFG signal from the lowermost layer has been reduced significantly less for layers 2 and 5 and not at all for layer 10. Furthermore, relatively strong methylene resonances can now be observed for the buried layers $(1,2$, and 5). For layer 10, no distinct $\mathrm{CH}_{2}$ resonance can be observed.

Tourillon et al. [200] combined SFG and a close-packed array of gold nanoparticles to probe, with a high sensitivity, the changes in conformation and orientation induced by the recognition process of avidin by biocytin. They first prepared a close-packed AuNP film on the aminophenyltrimethoxysilane monolayer on quartz. Then biocytinylated thiol (BioSH) was covalently linked to the surface of the AuNPs. This surface can then be used to probe the recognition process between biocytin and avidin by recording SFG spectra before and after the reaction. This approach represents a new platform with potential use in biosensors, diagnostics and bioactive layers. Moreover, the combination of SFG and a similarly prepared AuNP film can be used to probe adsorbed molecules $[159,204]$. Tourillon et al. [159] showed that when SFG is performed in a total internal reflection configuration combined with a dense AuNP monolayer, the conformation of adsorbed molecules can be studied with an excellent signal to noise ratio and high signal to background ratio. Dodecanethiol (DDT) was used as a 
probe molecule in order to assess the potentialities of the approach. Figure 15 shows the SFG spectra collected from a DDT SAM adsorbed on a dense AuNPs monolayer recorded in a total internal reflection configuration. The results show that a well-ordered SAM is formed on a dense AuNP monolayer in a similar manner to that obtained on plain gold where the methylene modes are typically less than $10 \%$ of the $\mathrm{CH}_{3}$ modes.

\section{Conclusion and outlook}

We have shown that three advanced optical spectroscopic techniques and their applications on various nanomaterialbased optical sensors modified with specific biomolecules represent a powerful detection platform for a broad range of biological molecules. Several key features set these biosensors apart from other sensor technologies available today: ultrahigh sensitivity, selectivity, and the potential for integration with other optical measurements. The examples described in this review show clearly the potential of these biosensors to significantly impact disease diagnosis, genetic screening, and drug delivery as well as serve as powerful new tools for research in many areas of biology.

Although some limitations of each technique exist, a combination of strengths and weakness from each technique will result in powerful analytical measurements. For example, optical parameters such as intensity, wavelength, polarization and fluorescence lifetime can be combined together to provide an overall and valuable information about the specimen. Hence, if fluorescence microscopy is integrated with other techniques with higher spatial resolution and lower optical diffraction limits, such as AFM and SEM, it will provide comprehensive information of samples with high morphology resolution while maintaining selectivity and sensitivity.

As for SFG, it is capable of providing a multitude of applications in surface systems concerning nanostructured

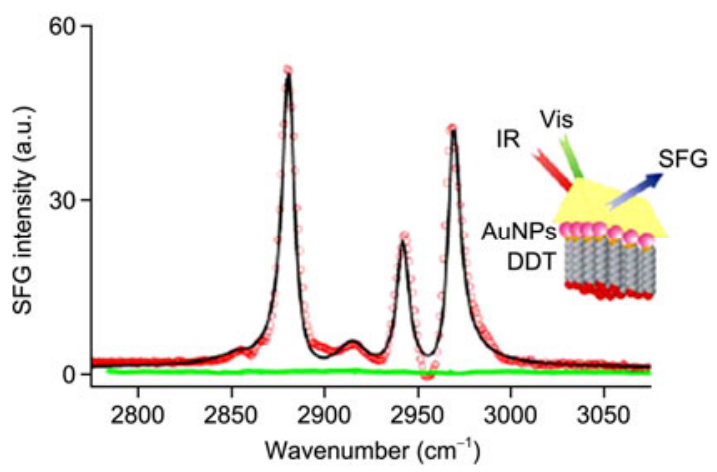

Figure 15 SFG spectra of a DDT SAM adsorbed on a dense AuNPs monolayer recorded in the TIR reflection configurations. The experimental data and the fitted curves are represented by open circles and lines, respectively. For comparison, the spectrum of the AuNPs monolayer is also shown (green line). Modified under permission of IOP from Ref. [159]. substances. Nano-sized biomacromolecules (peptides, proteins, DNA, and biofunctional synthetic polymers) and nanoparticles are promising research targets allowing us to explore the applicability of SFG to biomedicine, biosensors, and nano-optics/electronics. In systems containing nanoparticles, it is important to consider the SFG enhancement effect by the non-resonant surface plasmon signal generated from the nanoparticles. However, a fundamental understanding is still lacking regarding the effects of nanoparticle size, surface density, and surfactant coverage on the strength and resonant enhancement of the sum frequency signal. Even the enhancement mechanism itself still remains elusive. The exploration of the surface plasmon and the interference between the metal surface and the ordered substances can provide some interesting and important information on the surface chemistry. Studies on the noble metal nanoparticle/surfactant interface and semiconductor/surfactant interface not only have fundamental implications, but also have widespread industrial applications such as photo-voltaic cells, optical switching and nano-electronics. For fundamental research, it is important to explore the connection between the surface order and electron mobility in nanoelectronics. Finally, we believe that one of the future research directions is to probe some stimuli-induced (such as light, voltage, heat, and $\mathrm{pH}$ ) reorganization of the nanostructured surfaces by SFG.

1 Javey A, Guo J, Wang Q, et al. Ballistic carbon nanotube field-effect transistors. Nature, 2003, 424: 654-657

2 Zhang X X, Liu D F, Zhang L H, et al. Synthesis of large-scale periodic $\mathrm{ZnO}$ nanorod arrays and its blue-shift of UV luminescence. J Mater Chem, 2009, 19: 962-969

3 Huang M H, Mao S, Feick H, et al. Room-temperature ultraviolet nanowire nanolasers. Science, 2001, 292: 1897-1899

4 Cui Y, Lieber C M. Functional nanoscale electronic devices assembled using silicon nanowire building blocks. Science, 2001, 291: 851-853

5 Duan X F, Huang Y, Agarwal R, et al. Single-nanowire electrically driven lasers. Nature, 2003, 421: 241-245

6 Huang Y, Duan X F, Cui Y, et al. Logic gates and computation from assembled nanowire building blocks. Science, 2001, 294: 1313-1317

7 Li W Z, Xie S S, Qian L X, et al. Large-scale synthesis of aligned carbon nanotubes. Science, 1996, 274: 1701-1703

8 Yang Y C, Zhang X X, Gao M, et al. Nonvolatile resistive switching in single crystalline ZnO nanowires. Nanoscale, 2011, 3: 1917-1921

9 Wang S. Fundamentals of Semiconductor Theory and Device Physics. New York: Prentice Hall Englewood Cliffs, 1989

10 Gaponenko S V. Optical Properties of Semiconductor Nanocrystals. Cambridge: Cambridge University Press, 1998

11 Erickson H P. Size and shape of protein molecules at the nanometer level determined by sedimentation, gel filtration, and electron microscopy. Biol Proced Online, 2009, 11: 32-51

12 Dabbousi B, Rodriguez-Viejo J, Mikulec F V, et al. (CdSe)ZnS core-shell quantum dots: Synthesis and characterization of a size series of highly luminescent nanocrystallites. J Phys Chem B, 1997, 101: 9463-9475

13 Gerion D, Pinaud F, Williams S C, et al. Synthesis and properties of biocompatible water-soluble silica-coated $\mathrm{CdSe} / \mathrm{ZnS}$ semiconductor quantum dots. J Phys Chem B, 2001, 105: 8861-8871

14 Masuda H, Satoh M. Fabrication of gold nanodot array using anodic porous alumina as an evaporation mask. Jpn J Appl Phys, 1996, 35: L126-L129 
15 Fuhrmann B, Leipner H S, Höche H. Ordered arrays of silicon nanowiresproduced by nanosphere lithography and molecular beam epitaxy. Nano Lett, 2005, 5: 2524-2527

16 Liu D F, Xiang Y J, Zhang Z X, et al. Growth of ZnO hexagonal nanoprisms. Nanotechnology, 2005, 16: 2665-2669

17 Ma D D, Lee C S, Au F C, et al. Small-diameter silicon nanowire surfaces. Science, 2003, 299: 1874-1877

18 Stankovich S, Dikin D A, Piner R D, et al. Synthesis of graphenebased nanosheets via chemical reduction of exfoliated graphite oxide. Carbon, 2007, 45: 1558-1565

19 Geim A K, Novoselov K S. The rise of graphene. Nat Mater, 2007, 6: 183-191

20 Dikin D A, Stankovich S, Zimney E J, et al. Preparation and characterization of graphene oxide paper. Nature, 2007, 448: 457-460

21 Novoselov K, Geim A, Morozov S, et al. Electric field effect in atomically thin carbon films. Science, 2004, 306: 666-669

22 Zhang Z X, Yuan H J, Gao Y, et al. Large-scale synthesis and optical behaviors of $\mathrm{ZnO}$ tetrapods. Appl Phy Lett, 2007, 90: 15311611531163

23 Dick K A, Deppert K, Larsson M W, et al. Synthesis of branched "nanotrees" by controlled seeding of multiple branching events. Nat Mater, 2004, 3: 380-384

24 Zhao Y P, Ye D X, Wang G C, et al. Novel nano-column and nanoflower arrays by glancing angle deposition. Nano Lett, 2002, 2: 351-354

25 Tong Y H, Liu Y C, Shao C L, et al. Structural and optical properties of $\mathrm{ZnO}$ nanotower bundles. Appl Phy Lett, 2006, 88: 123111-123113

26 Chan W C W, Nie S M. Quantum dot bioconjugates for ultrasensitive nonisotopic detection. Science, 1998, 281: 2016-2018

27 Medintz I L, Uyeda H T, Goldman E R, et al. Quantum dot bioconjugates for imaging, labelling and sensing. Nat Mater, 2005, 4: 435-446

28 Han M, Gao X, Su J Z, et al. Quantum-dot-tagged microbeads for multiplexed optical coding of biomolecules. Nat Biotechnol, 2001, 19: 631-635

29 Cui Y, Wei Q Q, Park H K, et al. Nanowire nanosensors for highly sensitive and selective detection of biological and chemical species. Science, 2001, 293: 1289-1292

30 Li L S, Hu J T, Yang W D, et al. Band gap variation of size-and shape-controlled colloidal CdSe quantum rods. Nano Lett, 2001, 1: 349-351

31 Byun K M, Kim S J, Kim D. Design study of highly sensitive nanowire-enhanced surface plasmon resonance biosensors using rigorous coupled wave analysis. Opt Express, 2005, 13: 3737-3742

32 Lou J Y, Tong L M, Ye Z Z. Modeling of silica nanowires for optical sensing. Opt Express, 2005, 13: 2135-2140

33 Sun X M, Liu Z, Welsher K, et al. Nano-graphene oxide for cellular imaging and drug delivery. Nano Res, 2008, 1: 203-212

34 Mohanty N, Berry V. Graphene-based single-bacterium resolution biodevice and DNA transistor: Interfacing graphene derivatives with nanoscale and microscale biocomponents. Nano Lett, 2008, 8: 44694476

35 Wang $\mathrm{Y}, \mathrm{Li} \mathrm{Z} \mathrm{H}, \mathrm{Hu} \mathrm{D} \mathrm{H}$, et al. Aptamer/graphene oxide nanocomplex for in situ molecular probing in living cells. J Am Chem Soc, 2010, 132: 9274-9276

36 Shan C, Yang H, Song J, et al. Direct electrochemistry of glucose oxidase and biosensing for glucose based on graphene. Anal Chem, 2009, 81: 2378-2382

37 Yang P D, Yan R X, Fardy M. Semiconductor nanowire: What's next? Nano Lett, 2010, 10: 1529-1536

38 Allen B L, Kichambare P D, Star A. Carbon nanotube field-effecttransistor-based biosensors. Adv Mater, 2007, 19: 1439-1451

39 Patolsky F, Zheng G, Lieber C M. Nanowire-based biosensors. Anal Chem, 2006, 78: 4260-4269

40 Kapuscinski J. DAPI: A DMA-specific fluorescent probe. Biotech Histochem, 1995, 70: 220-233

41 James T W, Jope C. Visualization by fluorescence of chloroplast DNA in higher plants by means of the DNA-specific probe 4'6diamidino-2-phenylindole. J Cell Biol, 1978, 79: 623-630
42 Latt S A, Stetten G, Juergens L A, et al. Recent developments in the detection of deoxyribonucleic acid synthesis by 33258 Hoechst fluorescence. J Histochem Cytochem, 1975, 23: 493-505

43 Latt S, Stetten G. Spectral studies on 33258 Hoechst and related bisbenzimidazole dyes useful for fluorescent detection of deoxyribonucleic acid synthesis. J Histochem Cytochem, 1976, 24: 24-33

44 Liedberg B, Nylander C, Lunstrom I. Surface plasmon resonance for gas detection and biosensing. Sens Actuator, 1983, 4: 299-304

45 Homola J, Yee S S, Gauglitz G. Surface plasmon resonance sensors: Review. Sens Actuator B-Chem, 1999, 54: 3-15

46 Jönsson U, Fägerstam L, Ivarsson B, et al. Real-time biospecific interaction analysis using surface plasmon resonance and a sensor chip technology. Biotechniques, 1991, 11: 620-627

47 Kelly K L, Coronado E, Zhao L L, et al. The optical properties of metal nanoparticles: The influence of size, shape, and dielectric environment. J Phys Chem B, 2003, 107: 668-677

48 He L, Musick M D, Nicewarner S R, et al. Colloidal Au-enhanced surface plasmon resonance for ultrasensitive detection of DNA hybridization. J Am Chem Soc, 2000, 122: 9071-9077

49 Yuk J S, Ha K S. Proteomic applications of surface plasmon resonance biosensors: Analysis of protein arrays. Exp Mol Med, 2005, 37: $1-10$

50 Nath N, Chilkoti A. A colorimetric gold nanoparticle sensor to interrogate biomolecular interactions in real time on a surface. Anal Chem, 2002, 74: 504-509

51 Chah S, Hammond M R, Zare R N. Gold nanoparticles as a colorimetric sensor for protein conformational changes. Chem Biol, 2005, 12: 323-328

52 Chen Z, Shen Y R, Somorjai G A. Studies of polymer surfaces by sum frequency generation vibrational spectroscopy. Annu Rev Phys Chem, 2002, 53: 437-465

53 Chen Z. Understanding surfaces and buried interfaces of polymer materials at the molecular level using sum frequency generation vibrational spectroscopy. Polym Int, 2007, 56: 577-587

54 Chen Z. Investigating buried polymer interfaces using sum frequency generation vibrational spectroscopy. Prog Polym Sci, 2010, 35: 1376-1402

55 Liu Y W, Jasensky J, Chen Z. Molecularinteractions of proteins and peptides at interfaces studied by sum frequency generation vibrational spectroscopy. Langmuir, 2012, 28: 2113-2121

56 Ding B, Chen Z. Molecular interactions between cell penetrating peptide pep-1 and model cell membranes. J Phys Chem B, 2012, 116: 2545-2552

57 Wang J, Lee S H, Chen Z. Quantifying the ordering of adsorbed proteins in situ. J Phys Chem B, 2008, 112: 2281-2290

58 Wang J, Clarke M L, Zhang Y B, et al. Using isotope-labeled proteins and sum frequency generation vibrational spectroscopy to study protein adsorption. Langmuir, 2003, 19: 7862-7866

59 Even M A, Wang J, Chen Z. Structural information of mussel adhesive protein Mefp-3 acquired at various polymer/Mefp-3 solution interfaces. Langmuir, 2008, 24: 5795-5801

60 Le Clair S V, Nguyen K, Chen Z. Sum frequency generation studies on bioadhesion: Elucidating the molecular structure of proteins at interfaces. J Adhes, 2009, 85: 484-511

61 Nguyen K T, Le Clair S V, Ye S J, et al. Orientation determination of protein helical secondary structures using linear and nonlinear vibrational spectroscopy. J Phys Chem B, 2009, 113: 12169-12180

62 Wang J, Mark A, Chen X Y, et al. Detection of amide I signals of interfacial proteins in situ using SFG. J Am Chem Soc, 2003, 125: 9914-9915

63 Ye S J, Nguyen K T, Boughton A P, et al. Orientation difference of chemically immobilized and physically adsorbed biological molecules on polymers detected at the solid/liquid interfaces in situ. Langmuir, 2009, 26: 6471-6477

64 Ye S J, Nguyen K T, Chen Z. Interactions of alamethicin with model cell membranes investigated using sum frequency generation vibrational spectroscopy in real time in situ. J Phys Chem B, 2010, 114: 3334-3340

65 Chen X Y,Wang J, Sniadecki J J, et al. Probing $\alpha$-helical and $\beta$-sheet 
structures of peptides at solid/liquid interfaces with SFG. Langmuir, 2005, 21: 2662-2664

66 Ye S J, Nguyen K T, Clair S V L, et al. In situ molecular level studies on membrane related peptides and proteins in real time using sum frequency generation vibrational spectroscopy. J Struct Biol, 2009, 168: 61-77

67 Chen X Y, Boughton A P, Tesmer J J G, et al. In situ investigation of heterotrimeric $\mathrm{G}$ protein $\beta \gamma$ subunit binding and orientation on membrane bilayers. J Am Chem Soc, 2007, 129: 12658-12659

68 Chen X Y, Wang J, Boughton A P, et al. Multiple orientation of melittin inside a single lipid bilayer determined by combined vibrational spectroscopic studies. J Am Chem Soc, 2007, 129: $1420-1427$

69 Nguyen K T, Le Clair S V, Ye S J, et al. Molecular interactions between magainin 2 and model membranes in situ. J Phys Chem B, 2009, 113: 12358-12363

70 Chen X Y, Chen Z. SFG studies on interactions between antimicrobial peptides and supported lipid bilayers. Biochim Biophys Acta, 2006, 1758: 1257-1273

71 Nguyen K T, Soong R, Lm S C, et al. Probing the spontaneous membrane insertion of a tail-anchored membrane protein by sum frequency generation spectroscopy. J Am Chem Soc, 2010, 132: $15112-15115$

72 Avery C W, Palermo E F, Mclaughlin A, et al. Investigations of the interactions between synthetic antimicrobial polymers and substrate-supported lipid bilayers using sum frequency generation vibrational spectroscopy. Anal Chem, 2011, 83: 1342-1349

73 Avery $\mathrm{C} \mathrm{W}$, Chen Z. Characterizing the interactions between cell membranes and antimicrobials via sum-frequency generation vibrational spectroscopy. Antimicrobial Polymers, 2011, 429-457

74 Yang P, Ramamoorthy A, Chen Z. Membrane orientation of msi-78 measured by sum frequency generation vibrational spectroscopy. Langmuir, 2011, 27: 7760-7767

75 Boughton A P, Yang P, Tesmer V M, et al. Heterotrimeric G protein $\beta 1 \gamma 2$ subunits change orientation upon complex formation with $G$ protein-coupled receptor kinase 2 (GRK2) on a model membrane. Proc Natl Acad Sci USA, 2011, 108: E667-E673

76 Boughton A P, Nguyen K, Andricioaei I, et al. Interfacial orientation and secondary structure change in tachyplesin i: Molecular dynamics and sum frequency generation spectroscopy studies. Langmuir, 2011, 27: $14343-14351$

77 Asanuma $\mathrm{H}$, Noguchi $\mathrm{H}$, Uosaki $\mathrm{K}$, et al. Metal cation-induced deformation of dna self-assembled monolayers on silicon: Vibrational sum frequency generation spectroscopy. J Am Chem Soc, 2008, 130: 8016-8022

78 Walter S R, Geiger F M. DNA on stage: Showcasing oligonucleotides at surfaces and interfaces with second harmonic and vibrational sum frequency generation. J Phy Chem Lett, 2009, 1: 9-15

79 Sartenaer Y, Tourillon G, Dreesen L, et al. Sum-frequency generation spectroscopy of DNA monolayers. Biosens Bioelectron, 2007, 22: 2179-2183

80 Wang J, Chen X Y, Clarke M L, et al. Detection of chiral sum frequency generation vibrational spectra of proteins and peptides at interfaces in situ. Proc Natl Acad Sci USA, 2005, 102: 4978-4983

81 Nel A E, Mädler L,Velegol D, et al. Understanding biophysicochemical interactions at the nano-bio interface. Nat Mater, 2009, 8: $543-557$

82 Lodish H, Berk A, Zipursky S L, et al. Molecular Cell Biology. New York: W.H. Freeman \& Company, 1995

83 Mirkin C A, Letsinger R L, Mucic R C, et al. A DNA-based method for rationally assembling nanoparticles into macroscopic materials. Nature, 1996, 382: 607-609

84 Parak W J, Pellegrino T, Micheel C M, et al. Conformation of oligonucleotides attached to gold nanocrystals probed by gel electrophoresis. Nano Lett, 2003, 3: 33-36

85 Hanaki K, Momo A, Oku T, et al. Semiconductor quantum dot/ albumin complex is a long-life and highly photostable endosome marker. Biochem Biophys Res Commun, 2003, 302: 496-501

86 Erlanger B F, Chen B X, Zhu M, et al. Binding of an anti-fullerene
IgG monoclonal antibody to single wall carbon nanotubes. Nano Lett, 2001, 1: 465-468

87 Mahtab R, Harden H H, Murphy C J. Temperature-and saltdependent binding of long DNA to protein-sized quantum dots: Thermodynamics of "inorganic protein"-DNA interactions. J Am Chem Soc, 2000, 122: 14-17

88 Mattoussi H, Mauro J M, Goldman E R, et al. Self-assembly of $\mathrm{CdSe}-\mathrm{ZnS}$ quantum dot bioconjugates using an engineered recombinant protein. J Am Chem Soc, 2000, 122: 12142-12150

89 Mattoussi H, Mauro J, Goldman E, et al. Bioconjugation of highly luminescent colloidal cdse-zns quantum dots with an engineered two- domain recombinant protein. Phys Status Solidi B, 2001, 224: 277-283

90 Dubertret B, Skourides P, Norris D J, et al. In vivo imaging of quantum dots encapsulated in phospholipid micelles. Science, 2002, 298: $1759-1762$

91 Wu X Y, Liu H J, Liu J Q, et al. Immunofluorescent labeling of cancer marker Her2 and other cellular targets with semiconductor quantum dots. Nat Biotechnol, 2002, 21: 41-46

92 Chan W C W, Maxwell D J, Gao X, et al. Luminescent quantum dots for multiplexed biological detection and imaging. Curr Opin Biotechnol, 2002, 13: 40-46

93 Miyawaki A. Visualization of the spatial and temporal dynamics of intracellular signaling. Dev Cell, 2003, 4: 295-305

94 Schröck E, Du Manoir S, Veldman T, et al. Multicolor spectral karyotyping of human chromosomes. Science, 1996, 273: 494-497

95 Leatherdale C, Woo W K, Mikulec F, et al. On the absorption cross section of CdSe nanocrystal quantum dots. J Phys Chem B, 2002, 106: 7619-7622

96 Shao Y Y, Wang J, Wu H, et al. Graphene based electrochemical sensors and biosensors: A review. Electroanalysis, 2010, 22: 10271036

97 Huang Y X, Dong X C, Shi Y M, et al. Nanoelectronic biosensors based on CVD grown graphene. Nanoscale, 2010, 2: 1485-1488

98 Bruchez Jr M, Moronne M, Gin P, et al. Semiconductor nanocrystals as fluorescent biological labels. Science, 1998, 281: 2013-2016

99 Derfus A M, Chan W C W, Bhatia S N. Intracellular delivery of quantum dots for live cell labeling and organelle tracking. Adv Mater, 2004, 16: 961-966

100 Gao X H, Cui Y Y, Levenson R M, et al. In vivo cancer targeting and imaging with semiconductor quantum dots. Nat Biotechnol, 2004, 22: 969-976

101 Mahtab R, Rogers J P, Murphy C J. Protein-sized quantum dot luminescence can distinguish between "straight", "bent", and "kinked" oligonucleotides. J Am Chem Soc, 1995, 117: 9099-9100

102 Mahtab R, Rogers J P, Singleton C P, et al. Preferential adsorption of a "kinked" DNA to a neutral curved surface: Comparisons to and implications for nonspecific DNA-protein interactions. J Am Chem Soc, 1996, 118: 7028-7032

103 Taton T A, Mirkin C A, Letsinger R L. Scanometric DNA array detection with nanoparticle probes. Science, 2000, 289: 1757-1760

104 Yablonovitch E. Inhibited spontaneous emission in solid-state physics and electronics. Phys Rev Lett, 1987, 58: 2059-2062

105 Vos W, Polman A. Optical probes inside photonic crystals. MRS Bull, 2001, 26: 642-646

106 Asher S A, Peteu S F, Reese C E, et al. Polymerized crystalline colloidal array chemical-sensing materials for detection of lead in body fluids. Anal Bioanal Chem, 2002, 373: 632-638

107 Reese C E, Asher S A. Photonic crystal optrode sensor for detection of $\mathrm{Pb}^{2+}$ in high ionic strength environments. Anal Chem, 2003, 75: 3915-3918

108 Asher S A, Alexeev V L, Goponenko A V, et al. Photonic crystal carbohydrate sensors: Low ionic strength sugar sensing. J Am Chem Soc, 2003, 125: 3322-3329

$109 \mathrm{Fu} \mathrm{A} \mathrm{H,} \mathrm{Gu} \mathrm{W} \mathrm{W,} \mathrm{Boussert} \mathrm{B,} \mathrm{et} \mathrm{al.} \mathrm{Semiconductor} \mathrm{quantum} \mathrm{rods} \mathrm{as}$ single molecule fluorescent biological labels. Nano Lett, 2007, 7 : $179-182$

110 Dhar S, Liu Z, Thomale J, et al. Targeted single-wall carbon nanotube-mediated $\mathrm{Pt}(\mathrm{IV})$ prodrug delivery using folate as a homing 
device. J Am Chem Soc, 2008, 130: 11467-11476

111 Liu Z, Cai W B, He L, et al. In vivo biodistribution and highly efficient tumour targeting of carbon nanotubes in mice. Nat Nanotechnol, 2006, 2: 47-52

112 Al Faraj A, Cieslar K, Lacroix G, et al. In vivo imaging of carbon nanotube biodistribution using magnetic resonance imaging. Nano Lett, 2009, 9: 1023-1027

113 Singh R, Pantarotto D, Mccarthy D, et al. Binding and condensation of plasmid DNA onto functionalized carbon nanotubes: Toward the construction of nanotube-based gene delivery vectors. J Am Chem Soc, 2005, 127: 4388-4396

114 Podesta J E, Al-Jamal K T, Herrero M A, et al. Antitumor activity and prolonged survival by carbon-nanotube-mediated therapeutic sirna silencing in a human lung xenograft model. Small, 2009, 5: 1176-1185

115 Meng J, Duan J, Kong H, et al. Carbon nanotubes conjugated to tumor lysate protein enhance the efficacy of an antitumor immunotherapy. Small, 2008, 4: 1364-1370

116 Lin S, Keskar G, Wu Y, et al. Detection of phospholipid-carbon nanotube translocation using fluorescence energy transfer. Appl Phys Lett, 2006, 89: 143111-143118

117 Didenko V V, Baskin D S. Horseradish peroxidase-driven fluorescent labeling of nanotubes with quantum dots. Biotechniques, 2006, 40: 295-302

118 Cheng J, Fernando K A S, Veca L M, et al. Reversible accumulation of PEGylated single-walled carbon nanotubes in the mammalian nucleus. ACS Nano, 2008, 2: 2085-2094

119 Jia F M, Wu L, Meng J, et al. Preparation, characterization and fluorescent imaging of multi-walled carbon nanotube-porphyrin conjugate. J Mater Chem, 2009, 19: 8950-8957

120 Yang R H, Tang Z W, Yan J L, et al. Noncovalent assembly of carbon nanotubes and single-stranded DNA: An effective sensing platform for probing biomolecular interactions. Anal Chem, 2008, 80: 7408-7413

121 Yang R H, Jin J Y, Chen Y, et al. Carbon nanotube-quenched fluorescent oligonucleotides: Probes that fluoresce upon hybridization. J Am Chem Soc, 2008, 130: 8351-8358

$122 \mathrm{Lu} \mathrm{C} \mathrm{H,} \mathrm{Yang} \mathrm{H} \mathrm{H,} \mathrm{Zhu} \mathrm{C} \mathrm{L,} \mathrm{et} \mathrm{al.} \mathrm{A} \mathrm{graphene} \mathrm{platform} \mathrm{for} \mathrm{sensing}$ biomolecules. Angew Chem Int Ed, 2009, 48: 4785-4787

123 He S J, Song B, Li D, et al. A graphene nanoprobe for rapid, sensitive, and multicolor fluorescent DNA analysis. Adv Funct Mater, 2010, 20: 453-459

124 Jang H, Kim Y K, Kwon H M, et al. A graphene-based platform for the assay of duplex-dna unwinding by helicase. Angew Chem Int Ed, 2010, 122: 5839-5843

125 Broude N E. Stem-loop oligonucleotides: A robust tool for molecular biology and biotechnology. Trends Biotechnol, 2002, 20: $249-256$

126 Tyagi S, Kramer F R. Molecular beacons: Probes that fluoresce upon hybridization. Nat Biotechnol, 1996, 14: 303-308

127 Venkatesan N, Seo Y J, Kim B H. Quencher-free molecular beacons: A new strategy in fluorescence based nucleic acid analysis. Chem Soc Rev, 2008, 37: 648-663

128 Tyagi S, Bratu D P, Kramer F R. Multicolor molecular beacons for allele discrimination. Nat Biotechnol, 1998, 16: 49-53

129 Song S P, Liang Z Q, Zhang J, et al. Gold-nanoparticle-based multicolor nanobeacons for sequence-specific dna analysis. Angew Chem Int Ed, 2009, 48: 8670-8674

130 Tang Z W, Wu H, Cort J R, et al. Constraint of DNA on functionalized graphene improves its biostability and specificity. Small, 2010, 6: 1205-1209

131 Wen Y Q, Xing F F, He S J, et al. A graphene-based fluorescent nanoprobe for silver(I) ions detection by using graphene oxide and a silver-specific oligonucleotide. Chem Commun, 2010, 46: 2596-2598

132 Zhang M, Yin B C, Tan W, et al. A versatile graphene-based fluorescence "on/off" switch for multiplex detection of various targets. Biosens Bioelectron, 2011, 26: 3260-3265

133 Rich R L, Myszka D G. Survey of the year 2007 commercial optical biosensor literature. J Mol Recognit, 2008, 21: 355-400
134 Roh S, Chung T, Lee B. Overview of the characteristics of microand nano-structured surface plasmon resonance sensors. Sensors, 2011, 11: 1565-1588

135 Willets K A, Van Duyne R P. Localized surface plasmon resonance spectroscopy and sensing. Annu Rev Phys Chem, 2007, 58: 267-297

136 Anker J N, Hall W P, Lyandres O, et al. Biosensing with plasmonic nanosensors. Nat Mater, 2008, 7: 442-453

137 Jensen T R, Malinsky M D, Haynes C L, et al. Nanosphere lithography: Tunable localized surface plasmon resonance spectra of silver nanoparticles. J Phys Chem B, 2000, 104: 10549-10556

138 Miller M M, Lazarides A A. Sensitivity of metal nanoparticle surface plasmon resonance to the dielectric environment. J Phys Chem B, 2005, 109: 21556-21565

139 Jung L S, Campbell C T, Chinowsky T M, et al. Quantitative interpretation of the response of surface plasmon resonance sensors to adsorbed films. Langmuir, 1998, 14: 5636-5648

140 Haes A J, Van Duyne R P. A nanoscale optical biosensor: Sensitivity and selectivity of an approach based on the localized surface plasmon resonance spectroscopy of triangular silver nanoparticles. J Am Chem Soc, 2002, 124: 10596-10604

141 Zhao J, Jensen L, Sung J H, et al. Interaction of plasmon and molecular resonances for rhodamine $6 \mathrm{G}$ adsorbed on silver nanoparticles. J Am Chem Soc, 2007, 129: 7647-7656

142 Haes A J, Zou S L, Zhao J, et al. Localized surface plasmon resonance spectroscopy near molecular resonances. J Am Chem Soc, 2006, 128: 10905-10914

143 Zhao J, Das A, Zhang X Y, et al. Resonance surface plasmon spectroscopy: Low molecular weight substrate binding to cytochrome P450. J Am Chem Soc, 2006, 128: 11004-11005

144 Mitchell J S, Wu Y, Cook C J, et al. Sensitivity enhancement of surface plasmon resonance biosensing of small molecules. Anal Biochem, 2005, 343: 125-135

145 Byun K M. Development of nanostructured plasmonic substrates for enhanced optical biosensing. J Opt Soc Korea, 2010, 14: 65-76

146 Lyon L A, Musick M D, Natan M J. Colloidal Au-enhanced surface plasmon resonance immunosensing. Anal Chem, 1998, 70: 5177-5183

147 Liu X, Sun Y, Song D Q, et al. Sensitivity-enhancement of wavelength-modulation surface plasmon resonance biosensor for human complement factor 4. Anal Biochem, 2004, 333: 99-104

148 Reinhard B M, Siu M, Agarwal H, et al. Calibration of dynamic molecular rulers based on plasmon coupling between gold nanoparticles. Nano Lett, 2005, 5: 2246-2252

149 Sönnichsen C, Reinhard B M, Liphardt J, et al. A molecular ruler based on plasmon coupling of single gold and silver nanoparticles. Nat Biotechnol, 2005, 23: 741-745

150 Liu G L, Yin Y, Kunchakarra S, et al. A nanoplasmonic molecular ruler for measuring nuclease activity and DNA footprinting. Nat Nanotechnol, 2006, 1: 47-52

151 Hall W P, Ngatia S N, Van Duyne R P. LSPR biosensor signal enhancement using nanoparticle-antibody conjugates. J Phys Chem C, 2011, 115: 1410-1414

152 Jain P K, El-Sayed M A. Surface plasmon coupling and its universal size scaling in metal nanostructures of complex geometry: Elongated particle pairs and nanosphere trimers. J Phys Chem C, 2008, 112: 4954-4960

153 Lee T H, Lee S W, Jung J, et al. Signal amplification by enzymatic reaction in an immunosensor based on localized surface plasmon resonance (LSPR). Sensors, 2010, 10: 2045-2053

154 Sagle L B, Ruvuna L K, Ruemmele J A, et al. Advances in localized surface plasmon resonance spectroscopy biosensing. Nanomedicine, 2011, 6: 1447-1462

155 Hall W P, Anker J N, Lin Y, et al. A calcium-modulated plasmonic switch. J Am Chem Soc, 2008, 130: 5836-5837

156 Fujieda R, Yang M. LSPR sensitivity improvement by using cnts/au nanoparticle for bioanalysis. Adv Mater Res, 2012, 403: 4411-4415

157 Zou W, Liu W W, Luo L M, et al. Detection of nitro explosives via LSPR sensitive silver clusters embedded in porous silica. J Mater Chem, 2012, 22: 12474-12478

158 Yonzon C R, Jeoung E, Zou S, et al. A comparative analysis of 
localized and propagating surface plasmon resonance sensors: The binding of concanavalin A to a monosaccharide functionalized selfassembled monolayer. J Am Chem Soc, 2004, 126: 12669-12676

159 Tourillon G, Dreesen L, Volcke C, et al. Total internal reflection sum-frequency generation spectroscopy and dense gold nanoparticles monolayer: A route for probing adsorbed molecules. Nanotechnology, 2007, 18: 415301-415307

160 Shen Y R. The Principles of Nonlinear Optics. New York: WileyInterscience, 1984. 575-580

161 Gracias D, Chen Z, Shen Y R, et al. Molecular characterization of polymer and polymer blend surfaces. Combined sum frequency generation surface vibrational spectroscopy and scanning force microscopy studies. Acc Chem Res, 1999, 32: 930-940

162 Shultz M J, Schnitzer C, Simonelli D, et al. Sum frequency generation spectroscopy of the aqueous interface: Ionic and soluble molecular solutions. Int Rev Phys Chem, 2000, 19: 123-153

163 Gopalakrishnan S, Liu D, Allen H C, et al. Vibrational spectroscopic studies of aqueous interfaces: Salts, acids, bases, and nanodrops. Chem Rev, 2006, 106: 1155-1175

164 Perry A, Neipert C, Space B, et al. Theoretical modeling of interface specific vibrational spectroscopy: Methods and applications to aqueous interfaces. Chem Rev, 2006, 106: 1234-1258

165 Moore F, Richmond G. Integration or segregation: How do molecules behave at oil/water interfaces? Acc Chem Res, 2008, 41: 739-748

166 Guyot-Sionnest P, Hunt J, Shen Y R. Sum-frequency vibrational spectroscopy of a Langmuir film: Study of molecular orientation of a two-dimensional system. Phys Rev Lett, 1987, 59: 1597-1600

167 Hunt J, Guyot-Sionnest P, Shen Y R. Observation of CH stretch vibrations of monolayers of molecules optical sum-frequency generation. Chem Phys Lett, 1987, 133: 189-192

168 Chen Z. Molecular structures of buried polymer interfaces and biological interfaces detected by sum frequency generation vibrational spectroscopy. Acta Phys Chim Sin, 2012, 28: 504-521

169 Chen Z, Ward R, Tian Y, et al. Interaction of fibrinogen with surfaces of end-group-modified polyurethanes: A surface-specific sum-frequency-generation vibrational spectroscopy study. J Biomed Mater Res, 2002, 62: 254-264

170 Mermut O, Phillips D C, York R L, et al. In situ adsorption studies of a 14-amino acid leucine-lysine peptide onto hydrophobic polystyrene and hydrophilic silica surfaces using quartz crystal microbalance, atomic force microscopy, and sum frequency generation vibrational spectroscopy. J Am Chem Soc, 2006, 128: 3598-3607

171 Phillips D C, York R L, Mermut O, et al. Side chain, chain length, and sequence effects on amphiphilic peptide adsorption at hydrophobic and hydrophilic surfaces studied by sum-frequency generation vibrational spectroscopy and quartz crystal microbalance. J Phys Chem C, 2007, 111: 255-261

172 York R L, Browne W K, Geissler P L, et al. Peptides adsorbed on hydrophobic surfaces-A sum frequency generation vibrational spectroscopy and modeling study. Israel J Chem, 2007, 47: 51-58

173 Weidner T, Breen N F, Li K, et al. Sum frequency generation and solid-state NMR study of the structure, orientation, and dynamics of polystyrene-adsorbed peptides. Proc Natl Acad Sci USA, 2010, 107: 13288-13293

174 Weidner T, Apte J S, Gamble L J, et al. Probing the orientation and conformation of $\alpha$-helix and $\beta$-strand model peptides on self-assembled monolayers using sum frequency generation and NEXAFS spectroscopy. Langmuir, 2009, 26: 3433-3440

175 Fu L, Ma G, Yan E C Y. In situ misfolding of human islet amyloid polypeptide at interfaces probed by vibrational sum frequency generation. J Am Chem Soc, 2010, 132: 5405-5412

176 Fu L, Wang Z G, Yan E C Y. Chiral vibrational structures of proteins at interfaces probed by sum frequency generation spectroscopy. Int $\mathrm{J}$ Mol Sci, 2011, 12: 9404-9425

177 Fu L, Liu J, Yan E C Y. Chiral sum frequency generation spectroscopy for characterizing protein secondary structures at interfaces. J Am Chem Soc, 2011, 133: 2545-2552

178 Jung S Y, Lim S M, Albertorio F, et al. The vroman effect: A molecular level description of fibrinogen displacement. J Am Chem
Soc, 2003, 125: 12782-12786

179 Chen X, Sagle L B, Cremer P S. Urea orientation at protein surfaces. J Am Chem Soc, 2007, 129: 15104-15105

180 Hall S A, Jena K C, Trudeau T G, et al. Structure of leucine adsorbed on polystyrene from nonlinear vibrational spectroscopy measurements, molecular dynamics simulations, and electronic structure calculations. J Phys Chem C, 2011, 115: 11216-11225

181 Wang J, Buck S M, Chen Z. The effect of surface coverage on conformation changes of bovine serum albumin molecules at the air-solution interface detected by sum frequency generation vibrational spectroscopy. Analyst, 2003, 128: 773-778

182 Wang J, Paszti Z, Mark A, et al. Measuring polymer surface ordering differences in air and water by sum frequency generation vibrational spectroscopy. J Am Chem Soc, 2002, 124: 7016-7023

183 Wang J, Buck S M, Chen Z. Sum frequency generation vibrational spectroscopy studies on protein adsorption. J Phys Chem B, 2002, 106: $11666-11672$

184 Wang J, Clarke M L, Chen X Y, et al. Molecular studies on protein conformations at polymer/liquid interfaces using sum frequency generation vibrational spectroscopy. Surf Sci, 2005, 587: 1-11

185 Chen X Y, Clarke M L, Wang J, et al. Sum frequency generation vibrational spectroscopy studies on molecular conformation and orientation of biological molecules at interfaces. Int J Mod Phys B, 2005, 19: 691-713

186 Nguyen $\mathrm{K} \mathrm{T}$, King $\mathrm{J} \mathrm{T}$, Chen Z. Orientation determination of interfacial $\beta$-sheet structures in situ. J Phys Chem B, 2010, 114: 82918300

187 Lee S H, Wang J, Krimm S, et al. Irreducible representation and projection operator application to understanding nonlinear optical phenomena: Hyper-raman, sum frequency generation, and four-wave mixing spectroscopy. J Phys Chem A, 2006, 110: 7035-7044

188 Ye S J, Li H C, Wei F, et al. Observing a model ion channel gating action in model cell membranes in real time in situ: Membrane potential change induced alamethicin orientation change. J Am Chem Soc, 2012, 134: 6237-6243

189 Clarke M L, Wang J, Chen Z. Conformational changes of fibrinogen after adsorption. J Phys Chem B, 2005, 109: 22027-22035

190 Wang J, Chen X Y, Clarke M L, et al. Vibrational spectroscopic studies on fibrinogen adsorption at polystyrene/protein solution interfaces: Hydrophobic side chain and secondary structure changes. J Phys Chem B, 2006, 110: 5017-5024

191 Chen X Y, Wang J, Paszti Z, et al. Ordered adsorption of coagulation factor XII on negatively charged polymer surfaces probed by sum frequency generation vibrational spectroscopy. Anal Bioanal Chem, 2007, 388: 65-72

192 Han X, Soblosky L, Slutsky M, et al. Solvent effect and timedependent behavior of c-terminus-cysteine-modified cecropin $\mathrm{p} 1$ chemically immobilized on a polymer surface. Langmuir, 2011, 27: 7042-7051

193 Weeraman C, Yatawara A K, Bordenyuk A N, et al. Effect of nanoscale geometry on molecular conformation: Vibrational sumfrequency generation of alkanethiols on gold nanoparticles. J Am Chem Soc, 2006, 128: 14244-14245

194 Bordenyuk A N, Weeraman C, Yatawara A, et al. Vibrational sum frequency generation spectroscopy of dodecanethiol on metal nanoparticles. J Phys Chem C, 2007, 111: 8925-8933

195 Kawai T, Neivandt D J, Davies P B. Sum frequency generation on surfactant-coated gold nanoparticles. J Am Chem Soc, 2000, 122: 12031-12032

196 Aliaga C, Tsung C K, Alayoglu S, et al. Sum frequency generation vibrational spectroscopy and kinetic study of 2-methylfuran and 2,5-dimethylfuran hydrogenation over $7 \mathrm{~nm}$ platinum cubic nanoparticles. J Phys Chem C, 2011, 115: 8104-8109

197 Yeganeh M S, Dougal S M, Silbernagel B G. Sum frequency generation studies of surfaces of high-surface-area powdered materials. Langmuir, 2006, 22: 637-641

198 Kweskin S, Rioux R, Habas S, et al. Carbon monoxide adsorption and oxidation on monolayer films of cubic platinum nanoparticles investigated by infrared-visible sum frequency generation vibrational 
spectroscopy. J Phys Chem B, 2006, 110: 15920-15925

199 Holman J, Ye S, Neivandt D J, et al. Studying nanoparticle-induced structural changes within fatty acid multilayer films using sum frequency generation vibrational spectroscopy. J Am Chem Soc, 2004, 126: 14322-14323

200 Tourillon G, Dreesen L, Volcke C, et al. Close-packed array of gold nanoparticles and sum frequency generation spectroscopy in total internal reflection: A platform for studying biomolecules and biosensors. J Mater Sci, 2009, 44: 6805-6810

201 Somorjai G. New model catalysts (platinum nanoparticles) and new techniques (SFG and STM) for studies of reaction intermediates and surface restructuring at high pressures during catalytic reactions.
Appl Surf Sci, 1997, 121: 1-19

202 Dellwig T, Rupprechter G, Unterhalt H, et al. Bridging the pressure and materials gaps: High pressure sum frequency generation study on supported Pd nanoparticles. Phys Rev Lett, 2000, 85: 776-779

203 Rupprechter G, Freund H J. Adsorbate-induced restructuring and pressure-dependent adsorption on metal nanoparticles studied by electron microscopy and sum frequency generation spectroscopy. Top Catal, 2000, 14: 3-14

204 Humbert C, Busson B, Abid J P, et al. Self-assembled organic monolayers on gold nanoparticles: A study by sum-frequency generation combined with UV-vis spectroscopy. Electrochim Acta, 2005, 50: $3101-3110$

Open Access This article is distributed under the terms of the Creative Commons Attribution License which permits any use, distribution, and reproduction in any medium, provided the original author(s) and source are credited. 